\title{
Modelling seeding and neuroanatomic spread of pathology in amyotrophic lateral sclerosis
}

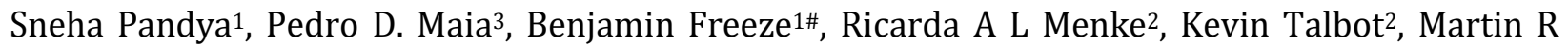
Turner $^{2 *}$ and Ashish Raj ${ }^{1,3^{*}}$

1Dept. of Radiology, Weill Cornell Medicine, 1300 York Avenue, New York, NY, USA

${ }^{2}$ Wellcome Centre for Integrative Neuroimaging \& Nuffield Department of Clinical Neurosciences, University of Oxford, Oxford, UK

${ }^{3}$ Dept. of Radiology and Biomedical Imaging, University of California, San Francisco, USA

\section{* Address correspondence to:}

Ashish Raj

Professor of Radiology and Bio-Engineering

185 Berry Street, Suite 370

University of California at San Francisco

San Francisco, CA 94121

\# Current address:

Department of Radiology

Massachusetts General Hospital

Harvard Medical School

Boston, MA
Prof Martin Turner

West Wing Level 6

John Radcliffe Hospital

Oxford OX2 7PZ

UK

Word count: 4912

Short title ( $<40$ characters): Seeding and progression in ALS 


\begin{abstract}
The neurodegenerative disorder amyotrophic lateral sclerosis (ALS) is characterized by the progressive loss of upper and lower motor neurons, with pathological involvement of cerebral motor and, additionally, extra-motor areas, in a clinicopathological spectrum with frontotemporal dementia (FTD). A key unresolved question is whether the distribution of pathology in ALS is driven by molecular factors such as regional gene expression, by differential network vulnerability, or is a combination of both. A system of histopathological staging of ALS based on the regional burden of TDP-43 pathology observed in post mortem brains has been supported to some extent by analysis of distribution of in vivo structural MRI changes. In this paper, computational modelling using a Network Diffusion Model (NDM) was used to investigate whether a process of focal pathological 'seeding' followed by structural network-based spread recapitulated post mortem histopathological staging and, secondly, whether this had any relationship to the pattern of expression of a panel of genes implicated in ALS across the healthy brain. Regionally parcellated T1-weighted MRI data from ALS patients (baseline $\mathrm{n}=79$ ) was studied in relation to a healthy control structural connectome and a database of associated regional cerebral gene expression. The NDM provided strong support for a structural network-based basis for regional pathological spread in ALS, but no simple relationship to the spatial distribution of ALS-related genes in the healthy brain. Intriguingly, the critical seed regions for spread within the model were not within the primary motor cortex but basal ganglia, thalamus and insula, where NDM recapitulated aspects of the post mortem histopathological staging system. Within the ALS-FTD clinicopathological spectrum, non-primary motor structures may be among the earliest sites of cerebral pathology.
\end{abstract}




\section{Introduction}

2 Amyotrophic lateral sclerosis (ALS), the commonest phenotype of motor neuron disease, is a 3 progressive and fatal neurodegenerative disorder with complex molecular underpinnings (Talbot et 4 al., 2018). The disease is clinically characterized by the progressive loss of upper motor neurons in 5 the primary motor cortex and corticospinal tract and lower motor neurons of the spinal cord and 6 brainstem. However ALS also involves extra-motor cerebral systems, with clear pathological, genetic 7 and clinical overlap with frontotemporal dementia (FTD) (Es et al., 2017), with the behavioral variant 8 being most common. Advances in neuroimaging have revealed many aspects of pathogenesis across the ALS-FTD spectrum (Turner et al., 2013; Chiò et al., 2014), with increasing interest in its potential to deliver therapeutic outcome measures (Menke et al., 2017).

11 The pattern of clinical symptom spread in ALS (Ravits et al., 2007b; Turner et al., 2010) and the associated spinal cord pathology (Ravits and La Spada, 2009), is not random but is focal in onset and spreads contiguously. Nearly all cases of ALS and around 50\% of FTD are associated with cytoplasmic neuronal and glial inclusions of aggregated 43kDa transactive response DNA-binding protein, TDP43 (Neumann et al., 2006). Post mortem histopathological classification has been interpreted as evidence of a stereotyped pattern of cerebral pathological involvement in ALS (Brettschneider et al., 2013). Several molecular mechanisms have been proposed to explain the apparent selective vulnerability of motor neurons. These include cell-autonomous factors, involving oxidative stress, excitotoxicity, and mitochondrial dysfunction (Turner et al., 2013) and non cell-autonomous factors involving cell-cell communication (e.g. glia (Philips and Rothstein, 2014)) or trans-neuronal transmission of aggregate-prone proteins through prion-like templating (Polymenidou and Cleveland, 2011; Riku, 2020), in which network connectivity might define the canonical pattern of spread (Seeley et al., 2009).

It is not yet clear how molecular vulnerability and network connectivity might combine in mediating regional patterns of pathology in ALS. Like other neurodegenerative diseases, the spatial topography of ALS histopathology is not related in a simple way to regional expression of genes implicated in 27 pathogenesis (Fusco et al., 1999; Jackson, 2014; Subramaniam, 2019). A high level of clinical and molecular heterogeneity in the ALS-FTD syndrome meanwhile hamper the ability to map its course in a precise manner to facilitate effective therapeutic trials (Turner and Swash, 2015).

30 In this paper we address these issues using computational modeling, gene expression analysis and 31 large observational imaging studies in ALS, combined with prior histopathological staging data. We 
1 interrogate whether focal seeding followed by structural network-based spread recapitulate

2 patterns of ALS pathology by employing a Network Diffusion Model (NDM) to map

3 neurodegenerative topography (Raj et al., 2012). This model was successful in recapitulating spatial

4 patterns of diverse proteinopathies including Alzheimer's (Raj et al., 2015), frontotemporal dementia

5 (Raj et al., 2012), Parkinson's disease (Freeze et al., 2018, 2019; Pandya et al., 2019), Huntington's

6 disease (Poudel et al., 2019) and progressive supranuclear palsy (Pandya et al., 2017).

Methods

\section{Participants}

Data used in this study were obtained after informed consent from participants in the longitudinal Oxford Study for Biomarkers in Motor Neuron Disease ('BioMOx') cohort based on referrals to a large tertiary ALS clinic and clinical assessment involving two experienced neurologists (KT, MRT). For the purposes of this group-level analysis, a diagnosis of ALS included those within all El Escorial clinical diagnostic categories at baseline (including those with pure upper or lower motor neuron syndromes clinically) who also showed clear progression of motor involvement in subsequent follow-up. Data were available for 79 such ALS participants (mean age at baseline 61 \pm 11 years, male:female 2:1, mean duration from symptom onset $49 \pm 57$ months). Of these, 48 were also able to undergo repeat MRI every 6 months to a maximum of 5 visits in total (cohort overlaps with (Menke et al., 2018)). One baseline participant initially labelled as ALS was removed from the study cohort later due to failure to progress. All ALS participants were apparently sporadic (i.e. no family history of ALS or FTD). The study predated routine genetic testing in the clinic, but subsequent experience predicts this would have identified up to 3 apparently sporadic ALS gene mutation carriers, which is not felt to be material to the outcome.

\section{Image acquisition and regional volumetric analysis}

24 Images were acquired using a 3T Siemens Trio scanner (Siemens AG) with a 12-channel head coil at the Oxford Centre for Clinical Magnetic Resonance (OCMR). A high resolution 3D MP-RAGE T1weighted sequence was obtained for each subject with the following parameters: 192 axial slices; repetition and echo time $(\mathrm{TR} / \mathrm{TE})=2040 / 4.7 \mathrm{~ms}$, flip angle $8^{\circ}, 1 \times 1 \times 1 \mathrm{~mm}^{3}$ voxel size, and $6 \mathrm{~min}$ acquisition time. 
total intracranial volume generated by FreeSurfer to correct for head size. Image processing steps

2 were visually inspected for white-gray matter boundary and skull-stripping errors to ensure they

3 had been carried out correctly. 6 subjects that rated either 'partial' or 'fail' due to FreeSurfer failure

4 or insufficient tissue contrast were excluded from analysis. A vector of regional atrophy was created

5 by using a two tailed $t$-test between ALS and normal mean ICV corrected regional volumes such that

$6 t_{\mathrm{ALS}}=\left\{t_{\mathrm{ALS}}(i) \mid i \in[1, N]\right\}(N=86)$. The $t$-statistic was converted to the natural positive range between

70 and 1 using the logistic transform given by $\Phi=1 /\left(1+\exp \left(-\left(t_{A L S}-\mathrm{a} 0\right) / \sigma / \operatorname{std}\left(t_{A L S}\right)\right)\right.$, where, $\sigma=2$ and $\mathrm{a} 0=0.5^{*} \sigma$ (Raj et al., 2015). This transformation maps t-values such that they asymptotically approach 0 as $t_{\mathrm{ALS}}$ approaches $-\infty$ and 1 as $t_{\mathrm{ALS}}$ approaches $+\infty$. The parameter $\sigma$ controls the steepness of the logistic function. These atrophy measures were then used to test the propagation modeling analyses using NDM.

\section{A healthy structural connectome}

Axial T1-weighted structural MRI scans using fast spoiled gradient-echo sequence(TE $=1.5 \mathrm{~ms}, \mathrm{TR}=$ $6.3 \mathrm{~ms}, \mathrm{TI}=400 \mathrm{~ms}, 15^{\circ}$ flip angle, $230 \times 230 \times 156$ isotropic $1 \mathrm{~mm}$ voxels) and high angular resolution diffusion tensor imaging data (DTI) (55 directions, $b=1,000 \mathrm{~s} / \mathrm{mm} 2,72$ 1.8-mm-thick interleaved slices, $0.8594 \mathrm{~mm} \times 0.8594 \mathrm{~mm}$ planar resolution) were acquired on a 3T GE Signa EXCITE scanner from fully consented 73 young healthy volunteers under a previous study approved by Weill Cornell's institutional review board (Kuceyeski et al., 2013). Thus, the cohort used to extract a healthy structural connectome was different from the 38 age-matched controls described earlier, that was used for determining regional atrophy. Probabilistic tractography was performed on the diffusion MRI data after seeding each voxel at the interface of the WM and GM boundary. The resulting streamlines were binned into subsets corresponding to every pair of GM regions given by the parcellation scheme described above. The anatomical connection strength (ACS), a measure of connectivity, was used in this paper (Iturria-Medina et al., 2007). ACS is defined as the weighted sum of the streamlines found to exist between any pair of gray matter structures, weighted by each streamline's probability score. The ACS is further normalized by a scaling factor equaling to the total sum of all streamlines. We define $c_{i, j}$ as the resulting connection strength between $i^{\text {th }}$ and $j^{\text {th }} \mathrm{GM}$ regions. We refer to the matrix collecting all pair-wise entries as the connectivity matrix $C=\left\{c_{i, j}\right\}$. Here the ACS is used as an approximation of the cross-sectional area of all axonal projections connecting two regions - a plausible choice given our goal of modeling the amount of pathology transmission conducted through these projections. Connections are assumed to be bidirectional since directionality is not deducible from DTI tractography data. 


\section{NDM for ALS pathology spread}

2 The hypothetical spread of disease-causing proteinopathy into the network represented by the connectivity matrix $C$ over time $t$ can be captured by starting a diffusion process from a "seed" region. Since we do not know a priori which region is the likely seed, we select every brain region as the seed region, one at a time (Raj et al., 2012). The overall strategy is to simulate a diffusion process on the connectivity graph for many time points, starting from each seed location, while recording its correlation with measured regional atrophy maps.

We modeled ALS progression as a diffusion process of the pathology load $\mathrm{x}$ on the graph $C$ over model time $t$. From (Raj et al., 2012) the transmission of pathology from region 1 to region 2 is represented as $\frac{d x_{1}}{d t}=\beta c_{1,2}\left(x_{2}-x_{1}\right)$ where $x_{1}$ and $x_{2}$ denote the extent of disease-causing pathology in each region, and $\beta$ is a global diffusivity constant. Denoting pathology from all regions $i$ into a vector $\mathbf{x}(t)=\left\{x_{i}(t)\right\}$, the above equation extends to become:

$$
\frac{d \mathbf{x}(t)}{d t}=-\beta H \mathbf{x}(t)
$$

where $H$ is the well-known graph Laplacian

$$
H=I-D^{-\frac{1}{2}} C D^{-\frac{1}{2}}
$$

where $\mathrm{D}$ is a diagonal matrix whose diagonal entries contain the degree of each node, degree being defined as the sum of weighted connections emanating from the node. Note, in order to accommodate regions having widely different out-degrees, we used the degree-normalized version of the Laplacian matrix (Raj et al., 2015). Eq. 1 admits a closed-form solution $\mathbf{x}(t)=e^{-\beta H t} \mathbf{x}_{\mathbf{0}}$ where $\mathbf{x}_{\mathbf{0}}$ is the initial pattern of the disease process at $t=0$, and we call term $e^{-\beta H t}$ the diffusion kernel since it acts essentially as a spatial and temporal blurring operator on $\mathbf{x}_{\mathbf{0}}$. The unit of the model's diffusion time $t$ is arbitrary (au). Global diffusivity $\beta$ is unknown, hence we chose a value that would roughly span ALS progression (3-10 years), giving $\beta=1$.

The NDM is described by pathology $\mathrm{x}(\mathrm{t})$ and our hypothesis is that it should correlate with empirical atrophy $\Phi$, Pearson correlation strength (R statistic) and p-values were calculated between the (static) empirical atrophy measured on the ALS group $\Phi$ and $\mathrm{x}(\mathrm{t})$ at all model timepoints $\mathrm{t}$.

Repeated seeding. The NDM was run for all 86 seed regions, each time starting from a different ROI, such that $\mathbf{x}_{\mathbf{0}}$ is a unit vector with 1 at the index of the seed and zeros at all other regions. We observed that the atrophy pattern in our group was generally bilateral, hence for repeated seeding 
1 experiments, we chose to seed bilaterally, so that two entries in the "unit" vector were assigned 1.

2 This was repeated for each region in turn, and the NDM-predicted pathology pattern was calculated.

3 For each predicted pathology vector $x^{i}(t)$ seeded at region $i$, the Pearson's correlation coefficient $\mathrm{R}$ occurring over all model timepoints $t$ was determined, giving $R^{i}(t)$. "R-t curves" were represented by plotting these $R^{i}(t)$ values on common axis. $R_{\text {max }}^{i}$ was recorded as the maximum value from each $R^{i}(t)$, which reflected the likelihood of i-th region as the true region of pathology onset.

Histopathological staging. We tested whether NDM recapitulated the post mortem histopathological staging published in ALS (Brettschneider et al., 2013), assigning each of the 86 regions available in our atlas an ALS stage from 1 to 4. Regions that were not part of this staging schema were arbitrarily assigned stage 5 , a category that denotes the least vulnerable regions X.

Spatiotemporal evolution from most likely seed regions. The top five regions $i$ with the highest $R_{\text {max }}^{i}$ were chosen to qualify as best seeds, along with the precentral gyrus which is considered a key early site of ALS. We tabulated a similar list of top 5 seed regions that gave the highest $R_{\max }$ (Pearson correlation) between the NDM and the ALS TDP-43 pathology staging data. An average of these two $R_{\max }$ values was computed to give a representation of $\mathrm{R}\left(R_{\text {avg }}\right)$ from both empirical atrophy and TDP-43 staging. The regions with the highest $R_{\text {avg }}$ were considered as the best seeds overall and were used as seeding locations for further analysis (see Results).

Regional gene expression analysis. Prominent genes linked to familial ALS were $(n=25)$ identified from various studies (Robberecht and Eykens, 2015; Smith et al., 2017; Vajda et al., 2017; Chia et al., 2018; Karch et al., 2018; Nicolas et al., 2018) and mapped to 86 regions in the Desikan-Killiany atlas as in (Freeze et al., 2018). Additionally, genes in which pathogenic variants have been associated with TDP-43 pathology $(n=26)$ (Scotter et al., 2015) were also mapped to 86 regions as above. A list of all the genes used in this study can be found in the supplementary data. For each gene, data was obtained from the publicly available human Allen Brain Atlas (ABA) (Hawrylycz et al., 2012). Briefly, the ABA includes 926 brain regions, with each region having microarray expression levels from a set of 58,692 probes that correspond to 21,245 distinct genes. Expression data for each of the 926 regions from ABA were mapped to the 86 regions of the Desikan-Killiany atlas. All samples for all probes within the same region were averaged and then normalized for each gene to produce a single expression value quantified as a z-score. White matter tracts were excluded from the analysis. Expression for each gene was averaged for six subject brains (which comprises data for 6 left hemispheres and 2 right hemispheres; more information can be found at help.brain-map.org/download/attachments/2818165/ Normalization_WhitePaper.pdf). 


\section{Statistical analyses}

2 Throughout this paper the primary test statistic used to evaluate all models was Pearson's correlation strength R. In each case the dependent variable was the vector of regional atrophy or ALS staging, while the dependent variables were the NDM-predicted regional vector, and/or regional gene expression. As described above, $\mathrm{R}$ was computed at each model time $\mathrm{t}$ and the highest value was chosen as the model evidence.

7 For the gene results, genes for each category were corrected for multiple comparisons using 8 Bonferroni method, with thresholded $\mathrm{p}_{\text {corr }}=0.05 / 25=0.002$ for ALS-related genes and thresholded $9 \mathrm{p}_{\text {corr }}=0.05 / 26=0.0019$ for TDP-43 specific genes. Correlation coefficients with $\mathrm{p}$-values less than $\mathrm{p}_{\text {corr }}$ were considered statistically significant. Next, $\mathrm{L}_{1}$ regularized regression model was created containing NDM from the seed region at $t_{\max }\left(\mathbf{x}_{\text {tmax }}^{\text {seed }}\right)$ and regional genetic expression profiles averaged across all subjects and probes. Ten-fold cross-validation was performed for each model across a range of values for the tuning parameter lambda $(\lambda)$ using the Matlab script 'lasso'. These mapped genes and $\mathbf{x}_{\text {tmax }}^{\text {seed }}$ were then correlated with the atrophy to achieve significant predictors for atrophy. Predictors for each lasso were corrected for multiple comparisons using Bonferroni method, with thresholded $\mathrm{p}_{\text {corr }}=0.05 /(1+25+26)=9.6 \times 10^{-4}$ for $\mathbf{x}_{\text {tmax }}^{\text {seed }}$, ALS-related genes, and TDP-43 specific genes. Correlation coefficients with $\mathrm{p}$-values less than $\mathrm{p}_{\text {corr }}$ were considered statistically significant.

Random scrambling. In order to build a null distribution for assigning significance to the NDM, we performed two levels of randomization experiments. 1) We ran the NDM on 2000 randomly scrambled versions of the connectivity matrix C. C was scrambled using a symmetric transformation of the network's nodes by randomly permuting entire rows and columns, and the NDM was evaluated for each shuffled network after bilateral Insula seeding. This scrambling procedure maintains the scrambled networks therefore constitute null or reference models which supplied significance values to results of the true model. 2) We ran the NDM on 2000 randomly scrambled ALS atrophy vector. Atrophy values in $t_{\text {norm }}$ vector were randomly assigned amongst the 86 cerebral regions with 2000 different permutations. This scrambling method maintained the true connectivity $\mathrm{C}$ but replaced true regional atrophy pattern with a random distribution of atrophy.

\section{Data availability}

All data used in this study will be made available upon reasonable request and relevant code will be 
1 Neuroimaging has an inherent commitment to data-sharing. To get access to the data and comply

2 with the research ethics committee approval an application to the corresponding author will be

3 required so that the precise geographical extent of sharing is known.

$4 \quad$ Results

5 Spatial distribution of ALS atrophy and repeated seeding of the NDM

6 Figure 1A shows glass-brain (LoCastro et al., 2014; Marinescu et al., 2019) illustrations of spatial

7 distribution of ALS atrophy from our cross-sectional cohort which is consistent with progression of

8 ALS pathology (Kassubek et al., 2005; Grosskreutz et al., 2006; Mezzapesa et al., 2007; Agosta et al.,

9 2010; Westeneng et al., 2015). Pathology in each region is proportional to the t-statistic of ALS

10 atrophy after logistic transform, where color towards red show increased severity. Table SI-1 shows

11 empirical atrophy values of top 20 regions averaged across both the hemispheres.

12 Each region was computationally seeded in succession and the NDM evolved over model time $t$ on 13 the healthy connectome $C$. The spatial distribution of $R_{\max }$, which is indicative of the likelihood of each region as a seed is depicted (Figure 1B). Figure 1C shows the distribution of empirical atrophy and $R_{\max }$ for each of these 86 regions alongside, which indicates that the NDM-derived seeding propensity value $\left(\mathrm{R}_{\max }\right)$ does not simply reproduce the regions displaying the highest atrophy but instead reflects the consequence of network transmission starting from that region.

Figure 1D shows the R-t curve revealing spread of $R_{\max }$ corresponding to the best fit between empirical data and the NDM seeded at the $\mathrm{i}^{\text {th }}$ region (Eq. 2). For each region, the R-t curve would yield an intermediate peak in R, resembling the best match between the NDM and empirical data, and then after diffusing uniformly with decreasing resemblance between the actual data and the NDM. Table SI-2 shows top 20 regions with maximum Pearson correlation strength for each region seeded in succession. Basal ganglia structures and the insula were among the top best seed regions for with the highest $R_{\text {avg }}$ as seen in Table 1.

To test whether the above results on group regional data and generic ALS staging data are also applicable to individual ALS subjects, we also ran NDM on ALS individuals and calculated maximum Pearson's R after seeding each of 43 bilateral ROIs for each subject. Peak R is achieved by frontal, 
bioRxiv preprint doi: https://doi.org/10.1101/2021.02.24.431118; this version posted February 25, 2021. The copyright holder for this preprint (which was not certified by peer review) is the author/funder, who has granted bioRxiv a license to display the preprint in perpetuity. It is made available under aCC-BY 4.0 International license.

from most subjects (Figure 1E). Thus, there is considerable inter-subject heterogeneity in seeding, while at the group level there is a convergence of the likely seeds in frontoinsular and BG regions.

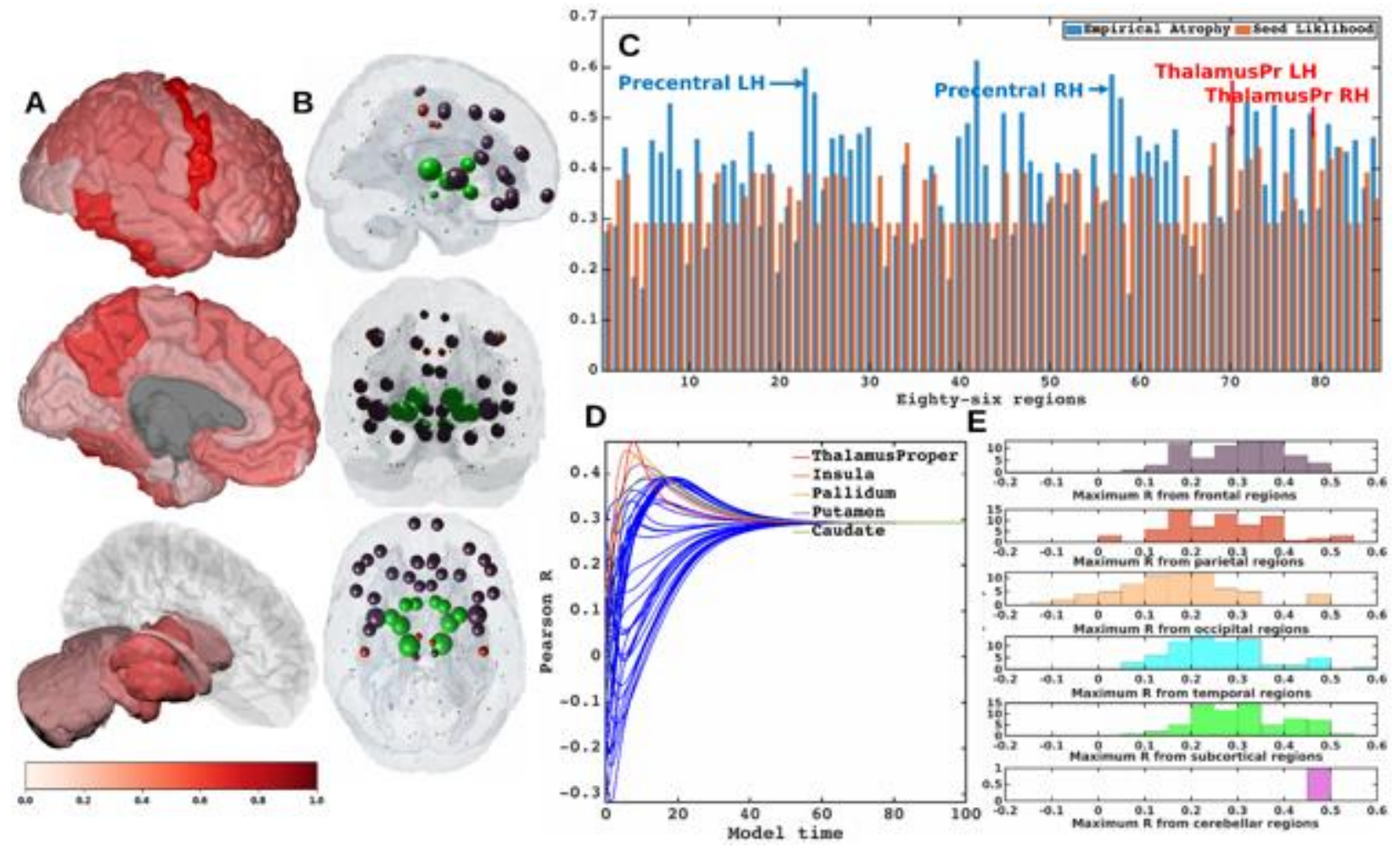

Figure 1: Spatial distribution of ALS atrophy and repeated seeding. A] Measured regional ALS atrophy are depicted by glass brain visualization. Bilateral volume loss was observed in somatosensory, frontotemporal, and subcortical regions, with most atrophy occurring in precentral gyrus, inferior temporal gyrus, precuneus, putamen, and thalamus regions. Severity of disease in each region is depicted in a color bar, where color towards red show increased severity. B] Each region was seeded in turn and NDM was played out for all time points. Pearson's $R$ was recorded at each time point between the model and ALS atrophy vector. As the diffusion time increases, more and more of the pathogenic agent escapes the seed region and enters the rest of the network. The point of maximum correlation with measured atrophy was recorded with glass brains of measured $R$ with spheres placed at the centroid of each brain region, and their diameter proportional to effect size. Spheres are color coded by lobe - frontal $=$ purple, parietal $=$ red, occipital $=$ orange, temporal $=$ cyan, subcortical $=$ green, and cerebellum = magenta. $C]$ Histogram of empirical atrophy and seed region likelihood as represented by $R_{\max }$ is shown side-by-side. Precentral which is the highest atrophied region when taking the average of empirical atrophy from left hemisphere $(\mathrm{LH})$ and right hemisphere $(\mathrm{RH})$ is not the best seed, thereby suggestive of inconsequential role of higher atrophy values in determination of $R_{\max }$. D] NDM seeded at bilateral regions indicates that the thalamus is the one of the most plausible candidate for ALS seeding - it has the highest peak $R$, and the characteristic intermediate peak indicative of true pathology spread. Other regions among the top five that obtained the highest $R$ were insula, pallidum, putamen, and caudate. $R$-t curves for the remaining regions are shown in blue. E] Histogram of maximum $R$ achieved from six major regions for all individual subjects. Rmax values were attained for each of these regions from 79 individual subjects. We can see that for most of the subjects' maximum $R$ (Rmax >0.4) was achieved from the frontal and subcortical regions compared to other regions. 
Table 1: List of regions most likely to serve as seeds of ALS pathology. Seeding likelihood of a region, shown in the rightmost column, is denoted by the average of the highest $R$ from both empirical atrophy and histopathological staging. Although the precentral gyrus does not have the highest seeding likelihood by these measures, it was included here due to its prominence as an early site in ALS.

\begin{tabular}{|c|c|c|c|c|}
\hline Region & Atrophy & $\mathbf{R}_{\max }$ (NDM vs Atrophy) & $\mathrm{R}$ (NDM vs ALS Staging) & Average R \\
\hline Insula & 0.41 & 0.45 & -0.64 & 0.55 \\
\hline Putamen & 0.51 & 0.42 & -0.65 & 0.53 \\
\hline Palladum & 0.48 & 0.44 & -0.61 & 0.53 \\
\hline Caudate & 0.32 & 0.40 & -0.52 & 0.46 \\
\hline Precentral & 0.59 & 0.39 & -0.42 & 0.40 \\
\hline Thalamus & 0.50 & 0.47 & -0.30 & 0.38 \\
\hline$R_{\text {max }}-$ Maximum Pearson Correlation & & \\
\hline
\end{tabular}

\section{Comparison with histopathological staging}

Table 2 shows ALS staging for TDP-43 pathology in each region. As shown in Figure 2 MRI atrophy and ALS staging are not highly correlated ( $\mathrm{R}=-0.27$ ), hence we wished to assess whether our model is also able to recapitulate post mortem histopathological staging. Therefore, the most likely seeding location for NDM was determined based on criteria that worked best for both atrophy and histopathological staging. Based on this criterion, the insula was selected as the best seed and used to play out NDM for all subsequent analyses (Table 1). Empirical atrophy, $\mathbf{x}_{\text {tmax }}^{\text {In }}$, and staging maps are shown in Figure 2A, 2B, and $2 \mathrm{C}$ respectively. Figure 2B shows the distribution of predicted atrophy as determined by $\mathbf{x}_{\text {tmax }}^{I n}$. Pathology severity in each region is proportional to the color gradient. Regions with maximum severity as indicated by the NDM corresponded to regions in the more advanced histopathological stages. Given that seeding from the thalamus (Th) consistently produced the best R against empirical data (Figure 1D, Table SI-2), results were also obtained from $\mathbf{x}_{\text {tmax }}^{T h}$ (Figure SI-1). This demonstrated bilateral volume loss mainly occurring in regions corresponding to advanced histopathological staging. 
bioRxiv preprint doi: https://doi.org/10.1101/2021.02.24.431118; this version posted February 25, 2021. The copyright holder for this preprint (which was not certified by peer review) is the author/funder, who has granted bioRxiv a license to display the preprint in perpetuity. It is made available under aCC-BY 4.0 International license.

1 Table 2: Post mortem histopathological stages for each of 43 bilateral regions

\begin{tabular}{|c|c|c|c|c|c|}
\hline Region Names & $\begin{array}{c}\text { ALS } \\
\text { Staging }\end{array}$ & Region Names & $\begin{array}{c}\text { ALS } \\
\text { Staging }\end{array}$ & Region Names & $\begin{array}{c}\text { ALS } \\
\text { Staging }\end{array}$ \\
\hline Bankssts & 5 & Parsorbitalis & 3 & CerebellumCortex & 5 \\
\hline Caudalanteriorcingulate & 3 & Parstriangularis & 3 & Thalamus_Proper & 3 \\
\hline Caudalmiddlefrontal & 2 & Pericalcarine & 5 & Caudate & 3 \\
\hline Cuneus & 5 & Postcentral & 3 & Putamen & 3 \\
\hline Entorhinal & 4 & Posteriorcingulate & 5 & Pallidum & 3 \\
\hline Fusiform & 5 & Precentral & 1 & Hippocampus & 4 \\
\hline Inferiorparietal & 5 & Precuneus & 5 & Amygdala & 4 \\
\hline Inferiortemporal & 5 & Rostralanteriorcingulate & 3 & Accumbensarea & 3 \\
\hline Isthmuscingulate & 5 & Rostralmiddlefrontal & 3 & Hypothalamus & 4 \\
\hline Lateraloccipital & 5 & Superiorfrontal & 2 & & \\
\hline Lateralorbitofrontal & 3 & Superiorparietal & 4 & & \\
\hline Lingual & 5 & Superiortemporal & 4 & & \\
\hline Medialorbitofrontal & 3 & Supramarginal & 4 & & \\
\hline Middletemporal & 4 & Frontalpole & 3 & & \\
\hline Parahippocampal & 4 & Temporalpole & 4 & & \\
\hline Paracentral & 3 & Transversetemporal & 4 & & \\
\hline Parsopercularis & 3 & Insula & 3 & & \\
\hline
\end{tabular}



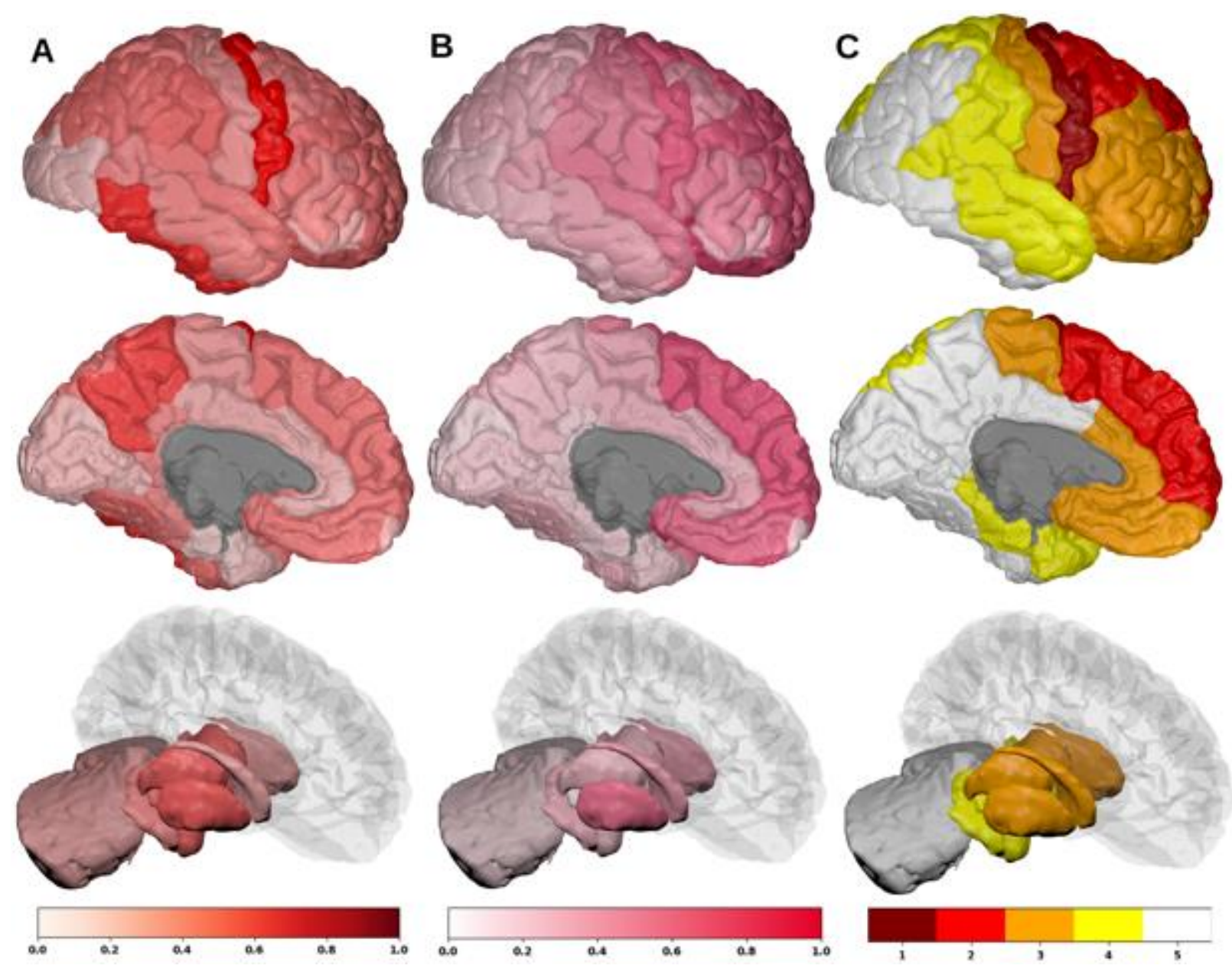

Figure 2: Spatial distribution of ALS atrophy, NDM Predicted atrophy and histopathological staging. A] Measured regional ALS atrophy are depicted by glass brain visualization. Bilateral volume loss was observed in somatosensory, frontotemporal, and subcortical regions, with most atrophy occurring in precentral gyrus, inferior temporal gyrus, precuneus, putamen, and thalamic regions. Severity of disease in each region is depicted in a color bar, where color towards red show increased severity. B] Glass brains of NDM seeded at the bilateral insula at $t_{\max }=6.06$ au yields progression of ALS from insula to connected, subcortical, anteromedial portions of temporal lobe and frontal areas. Bilateral volume loss is mainly observed in frontal and subcortical regions, with most atrophy occurring in later orbito-frontal, superior frontal, precentral, rostral middle-frontal, and putamen regions. Severity of disease in each region is depicted in a color bar, where color towards magenta showing increased severity. C] The ALS stage from 1-4 for each of the 43 bilateral regions. Stage 1 (maroon) starts with agranular motor cortex. The next affected regions (stage 2 in red) are the premotor cortex and parts of prefrontal neocortex. The pathology then progresses into striatum and into the prefrontal/postcentral cortices (stage 3 in orange), and finally to stage 4 show (yellow) involving anteromedial portions of the temporal lobe and the hippocampus. Stage 5 shows regions in white that are not part of the published histopathological staging system. 


\section{Spatiotemporal evolution of ALS atrophy}

2 The spatiotemporal evolution of ALS atrophy as recapitulated by network diffusion and evolved from

3 the insula at model times $t=2,4,6(a u)$ is shown in Figure 3. The evolution of network diffusion

4 process seeded at the insula starts at early stage $(t=2)$ through mature stage $(t=6)$, where the

5 maximum correspondence of NDM to empirical data occurred. Here, time is arbitrary, hence we have

6 used " $a u$ " as the unit of time for illustrative purpose only. At the initial stage, the disease involves

7 subcortical and frontal regions, followed by motor regions, and finally showing widespread

8 involvement of extra-motor and cortical regions. A linear positive association between empirical

9 atrophy and predicted atrophy $\left(\mathrm{R}=0.45, \mathrm{p}_{\mathrm{corr}}<5.8 \times 10^{-4}\right)$ as represented by the NDM at model times

$10 t=2,4,6$ (au) was demonstrated (Figure 3B). A linear association was found between the

11 histopathological staging and the NDM at model times $t=2,4,6$ (au) (Figure 3C), with a negative

12 correlation between the predicted atrophy and stage, which as model time progresses, shows

13 increased correlation between the NDM prediction from insula-seeding and each stage $(\mathrm{R}=-0.66$,

$14 \mathrm{p}_{\text {corr }}<5.8 \times 10^{-4}$ ). Similarly, we also explored spatiotemporal evolution from thalamic-seeding at

15 model times $t=3,5,8(\mathrm{au})$ (Figure SI-2). With thalamic-seeding, the predicted disease course involved

16 mainly subcortical regions at the initial stage, followed by diffusion into the frontal and motor

17 regions. 
A

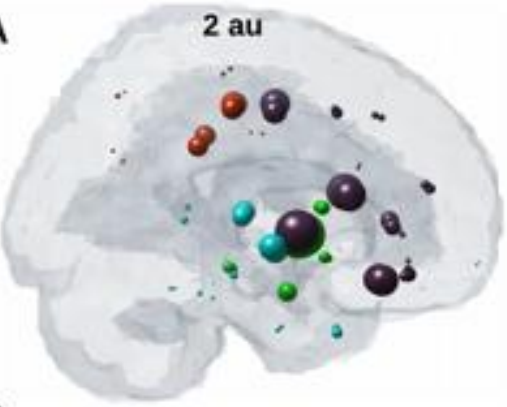

B

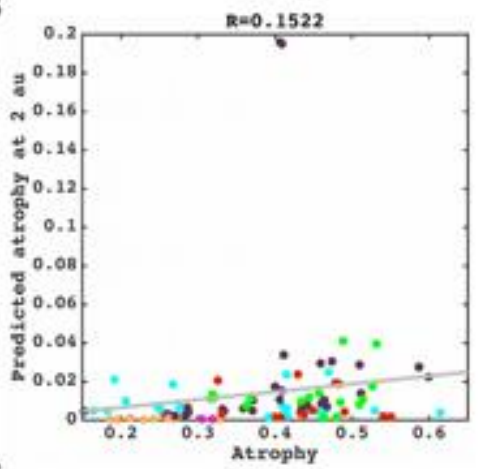

C

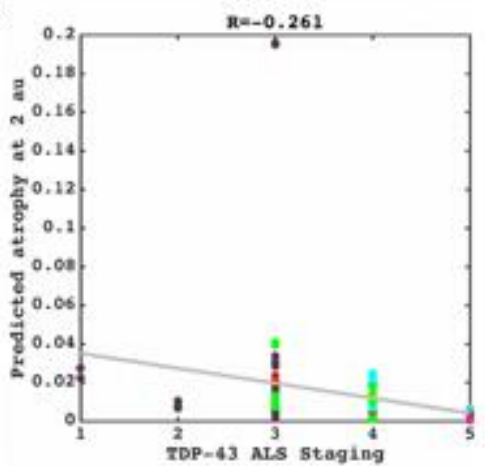

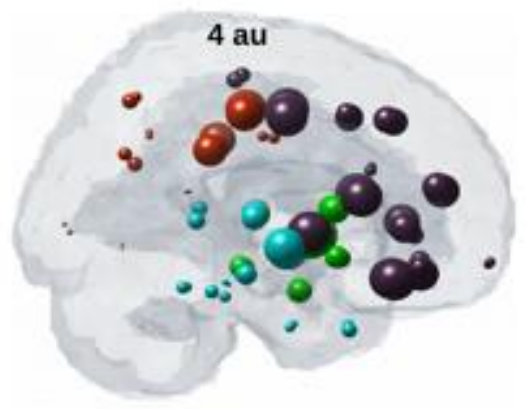
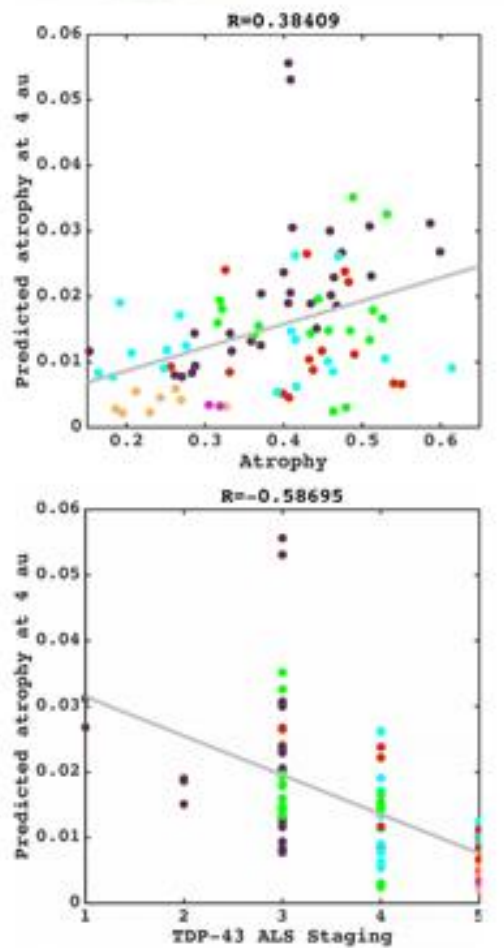
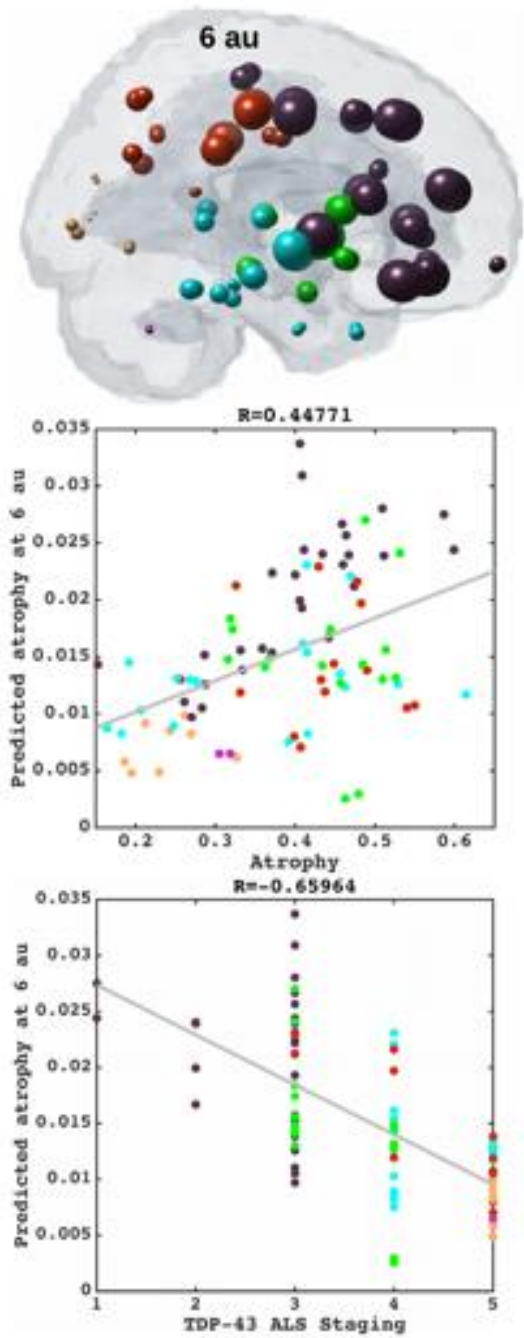

Figure 3: Spatiotemporal evolution, scatter plots of atrophy and histopathological staging with NDM at different model times. A] Evolution of insula-seeded network diffusion at model times $t=$ 2,4,6 (au) exhibited frontal involvement initially, followed by slower diffusion into the temporal, motor and subcortical cortices, and finally showing widespread involvement of cortical regions. This temporal sequencing predicted by the model suggest that volume loss in ALS involves extra-motor regions, particularly the prefrontal and subcortical regions. B] Scatter plot of NDM from insula versus empirical ALS atrophy at model times $t=2,4,6$ (au). Dots are color coded by lobe - frontal $=$ purple; parietal $=$ red; occipital = orange; temporal = cyan; subcortical = green; and cerebellum = magenta A positive correlation is observed between ALS empirical atrophy and NDM predicted atrophy from bilateral insula, which increases significantly $\left(R=0.45, p_{\text {corr }}<5.8 \times 10^{-4}, 0.05 / 86\right)$ at matured model times $t=$ 4,6 (au). C] Scatter plot of NDM from insula versus histopathological staging at model times $t=$ 2,4,6 (au). Dots are color coded by lobe - frontal = purple; parietal = red; occipital = orange; temporal = cyan; subcortical = green; and cerebellum = magenta . $A$ negative correlation was observed between the NDM and ALS staging from bilateral insula, which decreases significantly $\left(R=-0.66, p_{\text {corr }}<5.8 \times 10\right.$ 4) at matured model times $t=4,6(\mathrm{au})$. As time progressed, greater frontal, temporal and subcortical regions were involved with NDM closely resembling empirical ALS-FTD pathology. 


\section{Relationship of atrophy to regional ALS risk gene expression}

2 The linear relationship between different categories of genes (listed in Table SI-3) and empirical 3 atrophy was studied (Figure 4). Figure 4A shows distribution of empirical atrophy for reference.

4 Figures 4B and 4C show scatter plots of empirical atrophy versus averages of ALS-related genes, and

5 TDP-43 specific genes respectively. No association was found between ALS-related genes, TDP-43

6 specific genes empirical atrophy. Further, no association was found between atrophy and TARTDP gene itself (4D), which codes for TDP-43. Figures 4E, 4F, and 4G show local distribution of average of ALS-related genes, average of TDP-43 specific genes and TARDBP, respectively. Table SI-3 shows correlations of ALS-related genes, and their PCA vs. empirical atrophy (to the left) and correlations of TDP-43 specific genes and their PCA vs. empirical atrophy (to the right). These results suggest that genes alone do not contribute to regional vulnerability, and that linear association between atrophy and gene expression profiles are complex and cannot be explained by univariate analysis of genes alone.
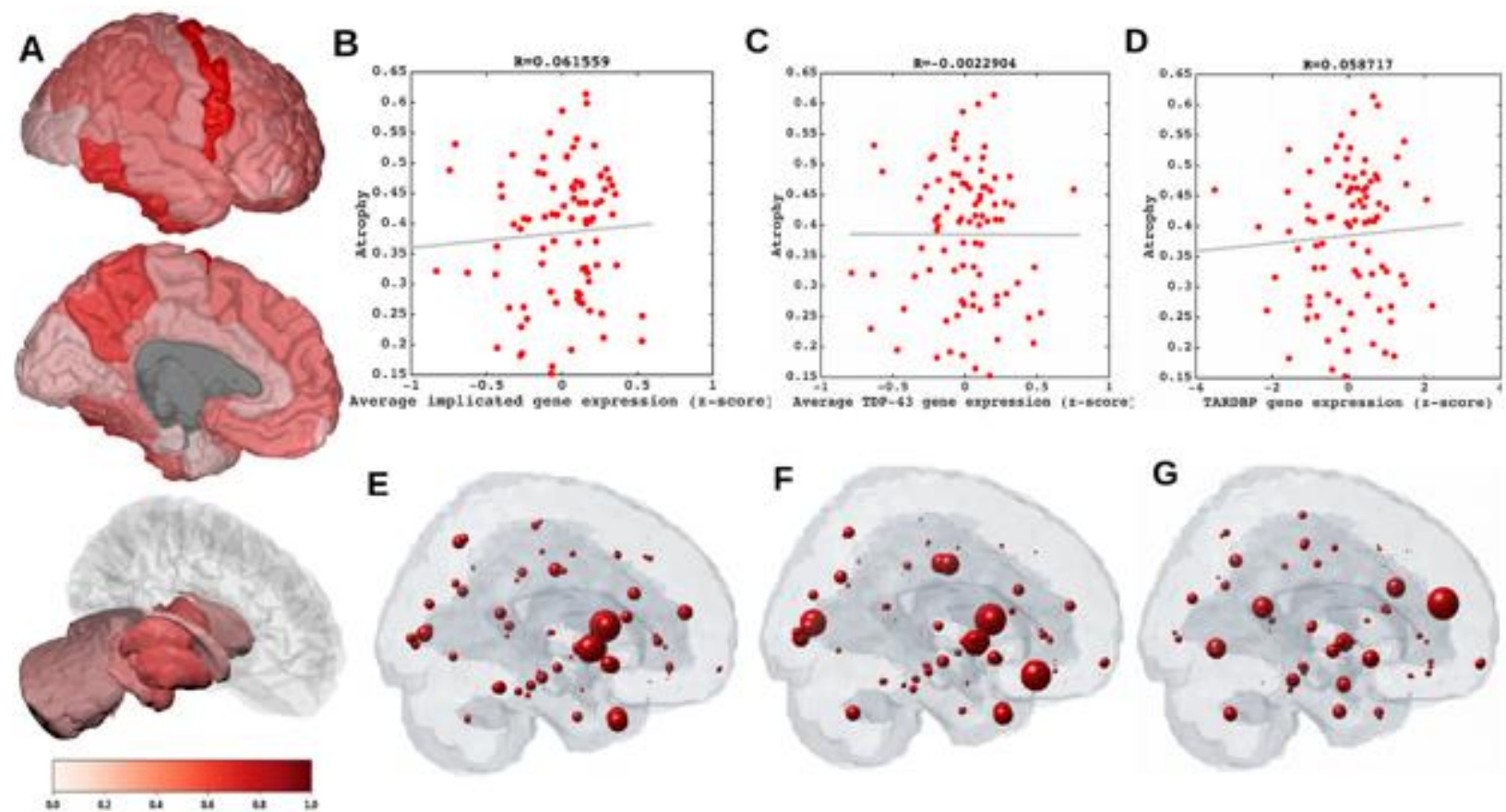

Figure 4: Spatial distribution of ALS atrophy, scatter plots of genes vs ALS atrophy, spatial distribution of genes. A] Measured regional ALS atrophy are depicted by glass brain visualization. Bilateral volume loss was observed in somatosensory, frontotemporal, and subcortical regions, with most atrophy occurring in precentral gyrus, inferior temporal gyrus, precuneus, putamen, and thalamic regions. Severity of disease in each region is depicted in a color bar, where color towards red show increased severity. B] Scatter plot of empirical atrophy vs average of all ALS-related genes shows no clear association. C] Scatter plot of empirical atrophy vs average of TDP-43 associated genes (F) shows no clear association. D] Scatter plot of empirical atrophy vs TARDBP gene expression (G) also shows no 
1 clear association - this was chosen for comparison, given that a small minority of ALS cases involve

2 mutations in TARDBP. Spheres in glass brains were placed at the centroid of each brain region, and their

3 diameter was proportional to effect size.

\section{$4 \quad$ NDM atrophy and regional ALS risk gene expression}

5 Although genes do not bear an association with regional atrophy directly, it is possible that they may

6 contribute to regional atrophy along with network transmission of pathology. To test this, we used

7 cross-validated L1 regularized regression (LASSO) feature selection to identify highly significant

8 predictors of ALS pathology from the NDM and ALS-related genes, and those genes that might result

9 in TDP-43 misfolding via downstream events. The model included the NDM predictor $\mathbf{x}_{\text {tmax }}^{\text {In }}, 25$ ALS-

10 related genes, and 26 TDP-43 specific genes (Figure 5). Six predictors survived lowest MSE in our

11 model as seen in Figure 5: $\mathbf{x}_{\text {tmax }}^{\text {In }}{ }^{* *}, \mathrm{CCNF}^{*}, \mathrm{UBQLN}^{*}$, PFN1*, and CSNK1E* with insula-seeding,

12 suggesting that the NDM is the best predictor of ALS atrophy, and that only few genes in addition to

13 the NDM contribute to the spatial pattern of disease, but in a weaker capacity.

14 Given that thalamic-seeding achieved the highest R from empirical data, LASSO analysis was repeated 15 with NDM from thalamic-seeding using $\mathbf{x}_{t \max }^{T h}$ and different categories of genes. With thalamic-

16 seeding of NDM, 10 predictors survived: $\mathbf{x}_{\text {tmax }}^{\text {Th }}{ }^{* *}$ KIF5A*, TBK1*, TIA1*, BNIP1*, CSNKIG1*,

17 CSNK2A1*, CSNK2A1P*, and CSNK2A2* (Figure SI-3). However, again genes were far less significant 18 contributors than NDM. 

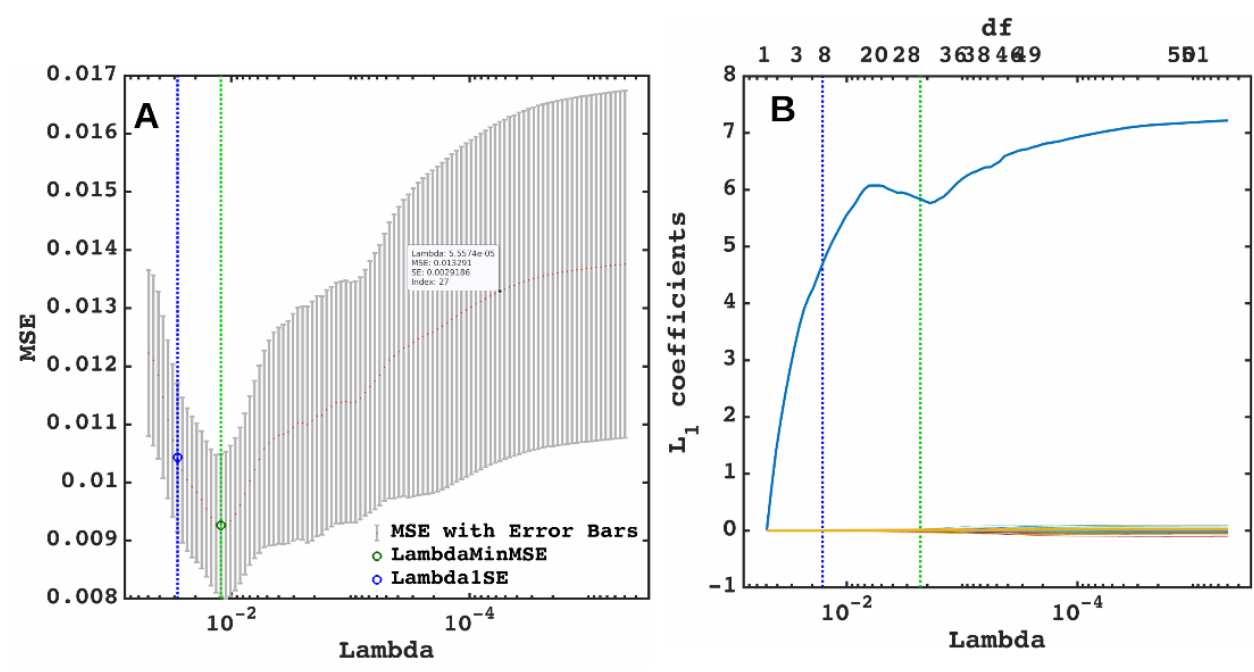

\begin{tabular}{|c|c|}
\hline ..... LambdaMinMSE & PFN1 \\
\hline ..... Lambda1SE & MAPT \\
\hline - NDMFromIN & BNIP1 \\
\hline KIF5A & TARDBP \\
\hline TNIP1 & UBQLN2 \\
\hline C9orf72 & - vCP \\
\hline TBK1 & - OPTN \\
\hline UNC13A & SQSTM1 \\
\hline C21orf 2 & ERBB4 \\
\hline CHCHD 10 & EGFR \\
\hline TUBA4A & QDPR \\
\hline CCNF & CSNK1G2 \\
\hline MATR3 & CSNK1G1 \\
\hline NEK1 & CSNK1A1L \\
\hline ANXA11 & CSNK1E \\
\hline TIA1 & - CSNK1A1 \\
\hline SOD1 & CSNK1D \\
\hline FUS & CSNK1G3 \\
\hline UBQLN2 & - CSNK1A1P1 \\
\hline DCTN1 & - CSNK2B \\
\hline ANG & CSNK2A1P \\
\hline TARDBP & CSNK2A1 \\
\hline VCP & CSNK2A2 \\
\hline OPTN & PARK2 \\
\hline SQSTM1 & UBE2E1 \\
\hline
\end{tabular}

C

\begin{tabular}{|c|c|c|c|c|}
\hline Region Names & Estimate & SE & tstat & p-val \\
\hline NDM & 6.85 & 1.77 & 3.87 & 0.0002 ** \\
\hline CCNF & 0.03 & 0.01 & 2.97 & 0.0040 * \\
\hline UBQLN2 & -0.02 & 0.01 & -2.08 & 0.0406 * \\
\hline PFN1 & 0.03 & 0.01 & 2.62 & 0.0107 * \\
\hline CSNK1E & -0.03 & 0.01 & -2.75 & 0.0074 * \\
\hline ** $\mathrm{p}_{\text {corr }}<9.6 \times 10^{-4} ;{ }^{*} \mathrm{p}<0.05$ & & & \\
\hline
\end{tabular}

Figure 5: Lasso plots and model parameters. A] Ten-fold cross validated MSE curves for determining regularized parameter lambda. Predictors with minimum L1 coefficient as a function of regularized parameter lambda with no more than one standard deviation (blue dotted line) were considered to be the most favorable. B] Cross-validated $L_{1}$ regularized regression coefficients as a function of tuning parameter lambda for a model containing the NDM from insula, ALS-related genes, and genes implicated in trans-synaptic TDP-43 transfer as predictors. Trace plot shows that as lambda increases towards the left, lasso sets various coefficients to zero, thereby removing them from the model. C] Model parameters and $p$-values of significant predictors that survived with $p<0.05$ (represented with "*”) and with Bonferroni corrected $p$ (represented with “**”). The NDM and expression profiles of CCNF, UBQLN2, PFN1, and CSNK1E have non-zero coefficients at minimum model MSE, indicating that these are essential predictive variables.

NDM evaluation against alternate modelling of network connectivity and ALS atrophy

To test the predictive power of NDM against alternate network models, we evaluated its specificity to ALS atrophy and to the connectome upon which it evolves. The distribution of Pearson's R over 2000 randomly simulated connectome matrices and atrophy vectors from insula and thalamicseeding are shown in Figure 6 and Figure SI-4 respectively. Random model's R was much lower than the maximum $\mathrm{R}$ of 0.45 from insula-seeding and 0.47 from thalamic-seeding which were achieved by the true model; statistically outside the $95 \%$ confidence interval, or $\mathrm{p}<0.05$. Hence, the reported insula and thalamic-seeded NDM outperforms all simulated models' prediction and is unlikely to be

21 explained by chance. 

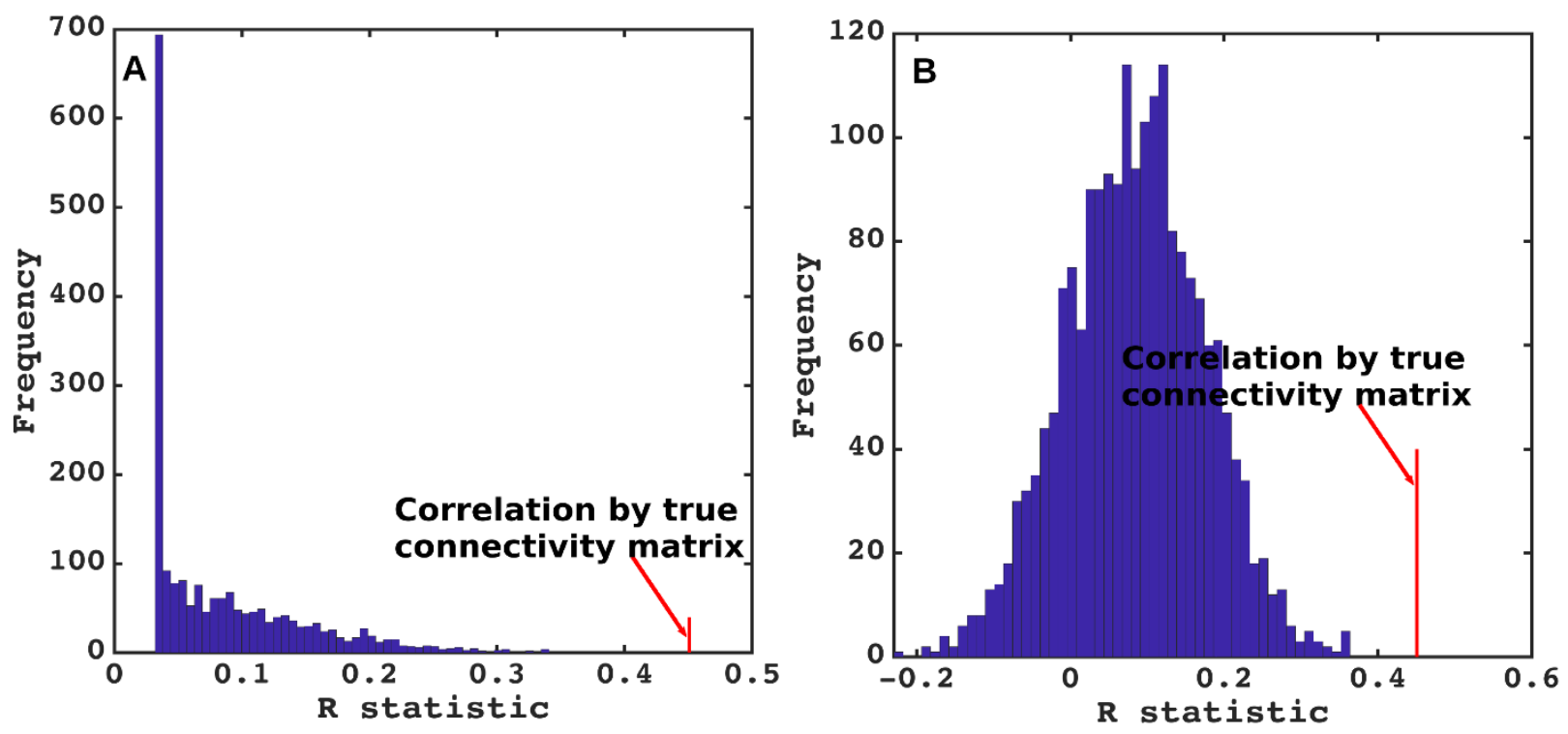

Figure 6: NDM evaluation against alternate models. A] Histogram of correlation strength between NDM and ALS data over 2000 shuffled networks. There is a hard limit on the left of this plot at R 0.03, which corresponds to the zero-diffusion time value of curve in Figure 1D. B] Histogram of correlation strength between NDM and 2000 shuffled ALS data over using unshuffled structural connectome. The true connectome was shuffled by symmetrically permuting its rows and columns randomly, and the NDM was evaluated for each shuffled network after bilateral insula-seeding. The best R achieved by each model was recorded and entered into the histogram. The null models are distributed well below the true model, indicating that the latter is highly unlikely to arise by chance $(p<0.05)$.

\section{Discussion}

Using a quantitative network-based model of pathology spread, this study sought to explore selective vulnerability and pathological progression in the ALS brain. We tested whether, setting each region of our brain atlas as the initiation site, the subsequent network spread modeled by the NDM correctly and significantly recapitulates cross-sectional patterns of regional atrophy and post mortem pathology staging. We also incorporated in our model the regional expression of ALS-related genes.

The results support structural network-based transmission in relation to regional atrophy, but with no significant relationship to the spatial distribution of the regional expression of ALS-related genes. Intriguingly, the critical seed regions for spread within the model were not within the primary motor cortex but in basal ganglia, thalamus and insula. NDM applied to these seed regions also recapitulated the post mortem histopathological staging system. Within a continuous ALS-FTD clinicopathological spectrum, these non-primary motor structures may be the sites of some of the earliest cerebral pathology. 


\section{Stereotyped Models of anatomical spread in ALS}

2 The focality of initial symptom onset and the non-random, typically regionally contiguous spread of 3 symptoms in ALS was shown to be mirrored, for limb involvement at least, by spinal cord 4 histopathology (Ravits et al., 2007a, b). These data were used to infer a theoretical model of 5 simultaneous cerebral focal onset and spread (Ravits and La Spada, 2009), but remain unproven. The 6 most consistent regions of cerebral pathological involvement in ALS have been the corticospinal tract and corpus callosum (Filippini et al., 2010; Müller et al., 2016), but with wider extra-motor involvement at baseline and a variable extent of both grey and white matter changes in longitudinal studies (Menke et al., 2014).

Post mortem studies defined a variably overlapping extent of TDP-43 pathology, arbitrarily divided into four "stages", with presumed but as yet unproven sequential trans-axonal progression in vivo (Brettschneider et al., 2013). In that model initial lesions were said to develop in the agranular motor cortex, in the bulbar and spinal somatomotor neurons, and the brainstem motor nuclei (stage 1). The next affected regions were the prefrontal neocortex, the brainstem reticular formation, the precerebellar nuclei, and the red nucleus (stage 2), then striatum and into the prefrontal/postcentral cortices (stage 3), finally involving anteromedial portions of the temporal lobe and the hippocampus (stage 4). This pathological staging has been supported by the same group in analysis of crosssectional in vivo MRI data (Gorges et al., 2018).

\section{Role of network transmission in ALS}

A plausible explanation for these patterns of progression may be trans-neuronal transmission of underlying pathology, which has been hypothesized in other neurodegenerative disorders including Alzheimer's Disease, Frontotemporal Dementia (FTD), Parkinson's Disease, Huntington's Disease and Creutzfeldt-Jakob disease (Spillantini et al., 1998; Lee et al., 2001; Neumann et al., 2006; Hansen et al., 2011; Herrera et al., 2011; Jack and Holtzman, 2013; Jucker and Walker, 2013; Walker et al., 2013; Maniecka and Polymenidou, 2015; Freeze et al., 2020). Concepts of seeding and self-templating of aberrant, aggregate-prone proteins have extended to ALS (Polymenidou and Cleveland, 2011), with a similar hypothesis of trans-neuronal transmission of pathogenic proteins between cells (Schmidt et al., 2016; Subramaniam, 2019). These emerging concepts in ALS and FTD were comprehensively reviewed recently (Riku, 2020). Broader concepts of structural and functional networks in health have been invoked for defining patterns of neurodegeneration (Seeley et al., 2009). MRI studies in ALS have supported the concept that structural connectivity mediates the 
spatial and temporal evolution of ALS atrophy and leads to network disintegration (Verstraete et al., 2011, 2014; Schmidt et al., 2016; Bede et al., 2018).

The present study is different from previous network studies in ALS because it does not investigate the question of whether structural connectivity networks themselves are being damaged by the disease process, but whether they serve as conduits for pathology transmission on the network. (Bede et al., 2018) found that the subcortical areas that undergo the highest cell loss in ALS are dictated by their connectivity to cortical regions. This could be due to network degeneration as above, but could also point to protein transmission along connections, as explored in our study.

\section{Insula, basal ganglia and thalamus as potential seeding sites in ALS}

The critical seed regions for widespread pathological spread within our model were not in the motor cortex but in basal ganglia, thalamus and insula. Furthermore, NDM applied to these seed regions recapitulated the post mortem TDP43-based histopathological staging scheme. These brain regions are well connected with prominent cortical areas undergoing atrophy in ALS (Bede et al., 2018). Basal ganglia involvement in ALS has been increasingly recognized (Bede et al., 2013; Riku, 2020) and the thalamus in particular has been shown to reflect the wider extent of cortical involvement in ALS (Chipika et al., 2020), notably in relation to the longitudinal spread of frontotemporal involvement (Tu et al., 2018).

A speculative interpretation of our results is that such sites may be common 'anchors' for what is a continuous ALS-FTD spectrum; see e.g. a recent review (Riku, 2020). The frontoinsula region appears to be one of the more selectively vulnerable and perhaps earliest sites of pathology in behavioral variant FTD, from which large von Economo neurons are prominently lost (Seeley et al., 2008; Kim et al., 2012). Similarly, the striatum is a site of early and prominent atrophy in bvFTD (Halabi et al.). Pathology in these non-primary motor deep gray matter structures may progress into either predominantly motor areas in ALS patients, or frontotemporal regions in FTD. Carriers of the intronic hexanucleotide expansion in C9orf72, the commonest inherited form of both ALS and FTD, tend to dichotomize into a phenotype with a predominance of one or other condition, even within the same pedigree (Mahoney et al., 2012), and the application of the methodology to a large cohort of such individuals might strengthen the hypothesis. 


\section{Limitations}

2 Our study was not able to accommodate many potential disease mechanisms like oxidative stress, 3 mitochondrial damage, metabolic dysregulation, and cell-type-specific factors, e.g. the specific role of 4 motoneurons. The NDM is a first-order, linear model of diffusive spread that assumes that the 5 structural connectivity network remains unchanged during disease course. Although all 6 neurodegenerative diseases lead to aberrant structural connectivity, in practice normative 7 connectomes as used here usually do not lead to significant reduction in the model's predictive power

8 (Powell et al, 2017). Individual subjects' genetic variables, medication history and age of symptom 9 onset were not analyzed. 


\section{ACKNOWLEDGEMENTS}

Authors convey grateful thanks to Chris Mezias and Justin Torok at Weill-Cornell, and Pablo Damasceno at UCSF for help with network and gene analysis.

\section{FUNDING}

This study was supported by NIH grants NS092802 and R01AG062196 (to AR). MRT was supported by the Medical Research Council \& Motor Neurone Disease Association Lady Edith Wolfson Fellowships (G0701923 \& MR/K01014X/1) and the Motor Neurone Disease Association Walker Professorship.

\section{COMPETING INTERESTS}

Authors report no competing interest. 


\section{Figure legends}

Figure 7: Spatial distribution of ALS atrophy and repeated seeding. A] Measured regional ALS atrophy are depicted by glass brain visualization. Bilateral volume loss was observed in somatosensory, frontotemporal, and subcortical regions, with most atrophy occurring in precentral gyrus, inferior temporal gyrus, precuneus, putamen, and thalamus regions. Severity of disease in each region is depicted in a color bar, where color towards red show increased severity. B] Each region was seeded in turn and NDM was played out for all time points. Pearson's R was recorded at each time point between the model and ALS atrophy vector. As the diffusion time increases, more and more of the pathogenic agent escapes the seed region and enters the rest of the network. The point of maximum correlation with measured atrophy was recorded with glass brains of measured $\mathrm{R}$ with spheres placed at the centroid of each brain region, and their diameter proportional to effect size. Spheres are color coded by lobe - frontal $=$ purple, parietal $=$ red, occipital $=$ orange, temporal $=$ cyan, subcortical $=$ green, and cerebellum $=$ magenta. $\mathrm{C}$ ] Histogram of empirical atrophy and seed region likelihood as represented by $R_{\max }$ is shown side-by-side. Precentral which is the highest atrophied region when taking the average of empirical atrophy from left hemisphere (LH) and right hemisphere $(\mathrm{RH})$ is not the best seed, thereby suggestive of inconsequential role of higher atrophy values in determination of $R_{\max }$. D] NDM seeded at bilateral regions indicates that the thalamus is the one of the most plausible candidate for ALS seeding - it has the highest peak $\mathrm{R}$, and the characteristic intermediate peak indicative of true pathology spread. Other regions among the top five that obtained the highest $\mathrm{R}$ were insula, pallidum, putamen, and caudate. R-t curves for the remaining regions are shown in blue. E] Histogram of maximum $\mathrm{R}$ achieved from six major regions for all individual subjects. Rmax values were attained for each of these regions from 79 individual subjects. We can see that for most of the subjects' maximum R (Rmax $>0.4$ ) was achieved from the frontal and subcortical regions compared to other regions.

Figure 8: Spatial distribution of ALS atrophy, NDM Predicted atrophy and histopathological staging. A] Measured regional ALS atrophy are depicted by glass brain visualization. Bilateral volume loss was observed in somatosensory, frontotemporal, and subcortical regions, with most atrophy occurring in precentral gyrus, inferior temporal gyrus, precuneus, putamen, and thalamic regions. Severity of disease in each region is depicted in a color bar, where color towards red show increased severity. B] Glass brains of NDM seeded at the bilateral insula at $t_{\max }=6.06$ au yields progression of ALS from insula to connected, subcortical, anteromedial portions of temporal lobe and frontal areas. Bilateral volume loss is mainly observed in frontal and subcortical regions, with most atrophy occurring in later orbito-frontal, superior frontal, precentral, rostral middle-frontal, and putamen regions. Severity of disease in each region is depicted in a color bar, where color towards magenta showing increased severity. C] The ALS stage from 1-4 for each of the 43 bilateral regions. Stage 1 (maroon) starts with agranular motor cortex. The next affected regions (stage 2 in red) are the premotor cortex and parts of prefrontal neocortex. The pathology then progresses into striatum and into the prefrontal/postcentral cortices (stage 3 in orange), and finally to stage 4 show (yellow) involving anteromedial portions of the temporal lobe and the hippocampus. Stage 5 shows regions in white that are not part of the published histopathological staging system.

Figure 9: Spatiotemporal evolution, scatter plots of atrophy and histopathological staging with NDM at different model times. A] Evolution of insula-seeded network diffusion at model times $t=2,4,6$ (au) exhibited frontal involvement initially, followed by slower diffusion into the temporal, motor and subcortical cortices, and finally showing widespread involvement of cortical regions. This 
temporal sequencing predicted by the model suggest that volume loss in ALS involves extra-motor regions, particularly the prefrontal and subcortical regions. B] Scatter plot of NDM from insula versus empirical ALS atrophy at model times $t=2,4,6(\mathrm{au})$. Dots are color coded by lobe - frontal = purple; parietal = red; occipital = orange; temporal = cyan; subcortical $=$ green; and cerebellum = magenta A positive correlation is observed between ALS empirical atrophy and NDM predicted atrophy from bilateral insula, which increases significantly $\left(\mathrm{R}=0.45, \mathrm{p}_{\text {corr }}<5.8 \times 10^{-4}, 0.05 / 86\right)$ at matured model times $t=4,6(\mathrm{au}) . \mathrm{C}]$ Scatter plot of NDM from insula versus histopathological staging at model times $t=2,4,6(a u)$. Dots are color coded by lobe - frontal = purple; parietal = red; occipital = orange; temporal = cyan; subcortical = green; and cerebellum = magenta. A negative correlation was observed between the NDM and ALS staging from bilateral insula, which decreases significantly $\left(\mathrm{R}=-0.66\right.$, $\mathrm{p}_{\text {corr }}$ $<5.8 \times 10^{-4}$ ) at matured model times $t=4,6(\mathrm{au})$. As time progressed, greater frontal, temporal and subcortical regions were involved with NDM closely resembling empirical ALS-FTD pathology.

Figure 10: Spatial distribution of ALS atrophy, scatter plots of genes vs ALS atrophy, spatial distribution of genes. A] Measured regional ALS atrophy are depicted by glass brain visualization. Bilateral volume loss was observed in somatosensory, frontotemporal, and subcortical regions, with most atrophy occurring in precentral gyrus, inferior temporal gyrus, precuneus, putamen, and thalamic regions. Severity of disease in each region is depicted in a color bar, where color towards red show increased severity. B] Scatter plot of empirical atrophy vs average of all ALS-related genes shows no clear association. C] Scatter plot of empirical atrophy vs average of TDP-43 associated genes (F) shows no clear association. D] Scatter plot of empirical atrophy vs TARDBP gene expression (G) also shows no clear association - this was chosen for comparison, given that a small minority of ALS cases involve mutations in TARDBP. Spheres in glass brains were placed at the centroid of each brain region, and their diameter was proportional to effect size.

Figure 11: Lasso plots and model parameters. A] Ten-fold cross validated MSE curves for determining regularized parameter lambda. Predictors with minimum L1 coefficient as a function of regularized parameter lambda with no more than one standard deviation (blue dotted line) were considered to be the most favorable. B] Cross-validated $\mathrm{L}_{1}$ regularized regression coefficients as a function of tuning parameter lambda for a model containing the NDM from insula, ALS-related genes, and genes implicated in trans-synaptic TDP-43 transfer as predictors. Trace plot shows that as lambda increases towards the left, lasso sets various coefficients to zero, thereby removing them from the model. C] Model parameters and p-values of significant predictors that survived with $\mathrm{p}<$ 0.05 (represented with “*”) and with Bonferroni corrected p (represented with “**”). The NDM and expression profiles of CCNF, UBQLN2, PFN1, and CSNK1E have non-zero coefficients at minimum model MSE, indicating that these are essential predictive variables.

Figure 12: NDM evaluation against alternate models. A] Histogram of correlation strength between NDM and ALS data over 2000 shuffled networks. There is a hard limit on the left of this plot at $\mathrm{R} \sim 0.03$, which corresponds to the zero-diffusion time value of curve in Figure 1D. B] Histogram of correlation strength between NDM and 2000 shuffled ALS data over using unshuffled structural connectome. The true connectome was shuffled by symmetrically permuting its rows and columns randomly, and the NDM was evaluated for each shuffled network after bilateral insula-seeding. The best $\mathrm{R}$ achieved by each model was recorded and entered into the histogram. The null models are distributed well below the true model, indicating that the latter is highly unlikely to arise by chance $(\mathrm{p}<0.05)$. 
Figure SI - 1: Spatial distribution of ALS atrophy, NDM Predicted atrophy and histopathological staging. A] Measured regional ALS atrophy are depicted by "glass brain" visualization. Bilateral volume loss is observed in somatosensory, frontotemporal, and subcortical regions, with most atrophy occurring in precentral gyrus, inferior temporal gyrus, precuneus, putamen, and thalamic regions. Severity of disease in each region is depicted in a color bar, where color towards red show increased severity. B] Glass brains of NDM seeded at the bilateral thalamus at $t_{\max }=8.08$ au yields progression of ALS from thalamus to connected extra-motor, subcortical and frontal areas. Bilateral volume loss is mainly observed in frontal and subcortical regions, with most atrophy occurring in insula, putamen, later orbito-frontal, superior frontal, and fusiform. Severity of disease in each region is depicted in a color bar, where color towards magenta showing increased severity. C] The ALS stage from 1-4 for each of the 43 bilateral regions. We can see that Stage 1 which is indicated by maroon starts with agranular motor cortex. The next affected regions (stage 2 in red) are the premotor cortex and parts of prefrontal neocortex. The pathology then progresses into striatum and into the prefrontal/postcentral cortices (stage 3 in orange), and finally to stage 4 show in yellow involving anteromedial portions of the temporal lobe and the hippocampus. Stage 5 denotes regions in white that are not part of ALS staging schema (i.e. outside the 4 accepted stages in ALS).

Figure SI - 2: Spatiotemporal evolution, scatter plots of atrophy and histopathological staging with NDM at different model times. A] Evolution of thalamic-seeded network diffusion at model times $t=3,5,8$ (au) exhibits subcortical areas as early affected regions, followed by somewhat slower diffusion into the motor and extra-motor cortices, specially prefrontal, and finally showing widespread involvement of cortical regions. This temporal sequencing predicted by the model suggest that volume loss in ALS involves extra-motor regions, particularly the prefrontal and subcortical regions. B] Scatter plot of NDM from thalamus versus empirical ALS atrophy at model times $t=3,5,8(\mathrm{au})$. Dots are color coded by lobe - frontal = purple; parietal = red; occipital = orange; temporal = cyan; subcortical $=$ green; and cerebellum = magenta . A positive correlation is observed between ALS empirical atrophy and NDM predicted atrophy from bilateral thalamus, which increases significantly $\left(\mathrm{R}=0.47, \mathrm{p}_{\text {corr }}<5.8 \times 10^{-4}, 0.05 / 86\right)$ at matured model times $t=5,8$ (au). As time progresses, more and more frontal, temporal and extra-motor regions are involved with NDM closely resembling ALS atrophy. C] Scatter plot of NDM from thalamus versus ALS staging of TDP-43 pathology at model times $t=3,5,8(\mathrm{au})$. Dots are color coded by lobe - frontal = purple; parietal = red; occipital = orange; temporal = cyan; subcortical = green; and cerebellum = magenta . A negative correlation is observed between the NDM and ALS staging from bilateral thalamus, which decreases significantly $\left(\mathrm{R}=-0.33, \mathrm{p}_{\text {corr }}<5.8 \times 10^{-4}\right)$ at matured model times $t=5,8(\mathrm{au})$. As time progresses, more and more frontal, temporal and subcortical regions are involved with NDM closely resembling empirical ALS atrophy.

Figure SI - 3: Lasso plots and model parameters. A] Ten-fold cross validated MSE curves for determining regularized parameter lambda. Predictors with minimum L1 coefficient as a function of regularized parameter lambda with no more than one standard deviation (blue dotted line) were considered to be the most favorable. B] Cross-validated $\mathrm{L}_{1}$ regularized regression coefficients as a function of tuning parameter lambda for a model containing the NDM from thalamus, ALS-related genes, and genes implicated in trans-synaptic TDP-43 transfer as predictors. Trace plot shows that's as lambda increases towards the left, lasso sets various coefficients to zero, thereby removing them from the model. C] Model parameters and p-values of significant predictors that survived with $\mathrm{p}<$ 0.05 (represented with “*”) and with Bonferroni corrected p (represented with “**”). The NDM and expression profiles of KIF5A, TBK1, TIA1, BNIP1, CSNK1G1, CSNKID, CSNK2A1, CSNK2A1P, and 
CSNK2A2 have non-zero coefficients at minimum model MSE, indicating that these are essential predictive variables.

Figure SI - 4: NDM evaluation against alternate models. A] Histogram of correlation strength between NDM and ALS data over 2000 shuffled networks. There is a hard limit on the left of this plot at $\mathrm{R} \sim 0.15$, which corresponds to the zero-diffusion time value of curve in Figure 1D. B] Histogram of correlation strength between NDM and 2000 shuffled ALS data over using unshuffled structural connectome. The true connectome was shuffled by symmetrically permuting its rows and columns randomly, and the NDM was evaluated for each shuffled network after bilateral thalamic-seeding. The best $\mathrm{R}$ achieved by each model was recorded and entered into the histogram. The null models are distributed well below the true model, indicating that the latter is highly unlikely to arise by chance $(p<0.05)$. 


\section{REFERENCES}

Agosta F, Chiò A, Cosottini M, De Stefano N, Falini A, Mascalchi M, et al. The Present and the Future of Neuroimaging in Amyotrophic Lateral Sclerosis. Am. J. Neuroradiol. 2010; 31: 1769-1777.

Bede P, Elamin M, Byrne S, McLaughlin RL, Kenna K, Vajda A, et al. Basal ganglia involvement in amyotrophic lateral sclerosis. Neurology 2013; 81: 2107-2115.

Bede P, Omer T, Finegan E, Chipika RH, Iyer PM, Doherty MA, et al. Connectivity-based characterisation of subcortical grey matter pathology in frontotemporal dementia and ALS: a multimodal neuroimaging study. Brain Imaging Behav. 2018; 12: 1696-1707.

Brettschneider J, Del Tredici K, Toledo JB, Robinson JL, Irwin DJ, Grossman M, et al. Stages of pTDP43 pathology in amyotrophic lateral sclerosis. Ann. Neurol. 2013; 74: 20-38.

Chia R, Chiò A, Traynor BJ. Novel genes associated with amyotrophic lateral sclerosis: diagnostic and clinical implications. Lancet. Neurol. 2018; 17: 94-102.

Chiò A, Pagani M, Agosta F, Calvo A, Cistaro A, Filippi M. Neuroimaging in amyotrophic lateral sclerosis: insights into structural and functional changes. Lancet. Neurol. 2014; 13: 1228-40.

Chipika RH, Finegan E, Li Hi Shing S, McKenna MC, Christidi F, Ming Chang K, et al. "Switchboard" malfunction in motor neuron diseases: selective pathology of thalamic nuclei in amyotrophic lateral sclerosis and primary lateral sclerosis. NeuroImage Clin. 2020: 102300.

Es M van, Hardiman 0, Chio A, Al-Chalabi A, Pasterkamp RJ, Veldink JJH, et al. Amyotrophic lateral sclerosis. Lancet 2017; 390: 2084-2098.

Filippini N, Douaud G, Mackay CE, Knight S, Talbot K, Turner MR. Corpus callosum involvement is a consistent feature of amyotrophic lateral sclerosis. Neurology 2010; 75: 1645-1652.

Freeze B, Acosta D, Pandya S, Zhao Y, Raj A. Regional expression of genes mediating trans-synaptic alpha-synuclein transfer predicts regional atrophy in Parkinson disease. NeuroImage Clin. 2018; 18: $456-466$.

Freeze B, Maia P, Pandya S, Raj A. Network mediation of pathology pattern in sporadic CreutzfeldtJakob disease [Internet]. Brain Commun. 2020Available from:

https://academic.oup.com/braincomms/advancearticle/doi/10.1093/braincomms/fcaa060/5837663 
Freeze B, Pandya S, Zeighami Y, Raj A. Regional transcriptional architecture of Parkinson's disease pathogenesis and network spread. Brain 2019; 142: 3072-3085.

Fusco FR, Chen Q, Lamoreaux WJ, Figueredo-Cardenas G, Jiao Y, Coffman JA, et al. Cellular localization of huntingtin in striatal and cortical neurons in rats: Lack of correlation with neuronal vulnerability in Huntington's disease. J. Neurosci. 1999; 19: 1189-1202.

Gorges M, Del Tredici K, Dreyhaupt J, Braak H, Ludolph AC, Müller H-P, et al. Corticoefferent pathology distribution in amyotrophic lateral sclerosis: in vivo evidence from a meta-analysis of diffusion tensor imaging data. Sci. Rep. 2018; 8: 15389.

Grosskreutz J, Kaufmann J, Frädrich J, Dengler R, Heinze H-J, Peschel T. Widespread sensorimotor and frontal cortical atrophy in Amyotrophic Lateral Sclerosis. BMC Neurol. 2006; 6: 17.

Halabi C, Halabi A, Dean DL, Wang P-N, Boxer AL, Trojanowski JQ, et al. Patterns of striatal degeneration in frontotemporal dementia. Alzheimer Dis. Assoc. Disord.; 27: 74-83.

Hansen C, Angot E, Bergström A-L, Steiner JA, Pieri L, Paul G, et al. $\alpha$-Synuclein propagates from mouse brain to grafted dopaminergic neurons and seeds aggregation in cultured human cells. J. Clin. Invest. 2011; 121: 715-25.

Hawrylycz MJ, Lein ES, Guillozet-Bongaarts AL, Shen EH, Ng L, Miller JA, et al. An anatomically comprehensive atlas of the adult human brain transcriptome. Nature 2012; 489: 391-399.

Herrera F, Tenreiro S, Miller-Fleming L, Outeiro TF. Visualization of cell-to-cell transmission of mutant huntingtin oligomers. PLoS Curr. 2011

Iturria-Medina Y, Canales-Rodríguez EJ, Melie-García L, Valdés-Hernández PA, Martínez-Montes E, Alemán-Gómez Y, et al. Characterizing brain anatomical connections using diffusion weighted MRI and graph theory. Neuroimage 2007; 36: 645-660.

Jack CR, Holtzman DM. Biomarker Modeling of Alzheimer's Disease. Neuron 2013; 80: 1347-1358.

Jackson WS. Selective vulnerability to neurodegenerative disease: The curious case of Prion Protein. DMM Dis. Model. Mech. 2014; 7: 21-29.

Jucker M, Walker LC. Self-propagation of pathogenic protein aggregates in neurodegenerative diseases. Nature 2013; 501: 45-51.

Karch CM, Wen N, Fan CC, Yokoyama JS, Kouri N, Ross OA, et al. Selective Genetic Overlap Between 
Amyotrophic Lateral Sclerosis and Diseases of the Frontotemporal Dementia Spectrum. JAMA Neurol. 2018; 75: 860-875.

Kassubek J, Unrath A, Huppertz H, Lulé D, Ethofer T, Sperfeld A, et al. Global brain atrophy and corticospinal tract alterations in ALS, as investigated by voxel-based morphometry of 3-D MRI. Amyotroph. Lateral Scler. 2005; 6: 213-220.

Kim E-J, Sidhu M, Gaus SE, Huang EJ, Hof PR, Miller BL, et al. Selective frontoinsular von Economo neuron and fork cell loss in early behavioral variant frontotemporal dementia. Cereb. Cortex 2012; 22: 251-9.

Kuceyeski A, Maruta J, Relkin N, Raj A. The Network Modification (NeMo) Tool: elucidating the effect of white matter integrity changes on cortical and subcortical structural connectivity. Brain Connect. 2013; 3: 451-63.

Lee VM-Y, Goedert M, Trojanowski JQ. Neurodegenerative Tauopathies. Annu. Rev. Neurosci. 2001; 24: 1121-1159.

LoCastro E, Kuceyeski A, Raj A. Brainography: An Atlas-Independent Surface and Network Rendering Tool for Neural Connectivity Visualization. Neuroinformatics 2014; 12: 355-359.

Mahoney CJ, Beck J, Rohrer JD, Lashley T, Mok K, Shakespeare T, et al. Frontotemporal dementia with the C90RF72 hexanucleotide repeat expansion: clinical, neuroanatomical and neuropathological features. Brain 2012; 135: 736-750.

Maniecka Z, Polymenidou M. From nucleation to widespread propagation: A prion-like concept for ALS. Virus Res. 2015; 207: 94-105.

Marinescu R V., Eshaghi A, Alexander DC, Golland P. BrainPainter: A Software for the Visualisation of Brain Structures, Biomarkers and Associated Pathological Processes. 2019. p. 112-120.

Menke RAL, Agosta F, Grosskreutz J, Filippi M, Turner MR. Neuroimaging Endpoints in Amyotrophic Lateral Sclerosis. Neurotherapeutics 2017; 14: 11-23.

Menke RAL, Körner S, Filippini N, Douaud G, Knight S, Talbot K, et al. Widespread grey matter pathology dominates the longitudinal cerebral MRI and clinical landscape of amyotrophic lateral sclerosis. Brain 2014; 137: 2546-55.

Menke RAL, Proudfoot M, Talbot K, Turner MR. The two-year progression of structural and functional cerebral MRI in amyotrophic lateral sclerosis. NeuroImage Clin. 2018; 17: 953-961. 
Mezzapesa DM, Ceccarelli A, Dicuonzo F, Carella A, De Caro MF, Lopez M, et al. Whole-brain and regional brain atrophy in amyotrophic lateral sclerosis. AJNR. Am. J. Neuroradiol. 2007; 28: 255-9.

Müller H-P, Turner MR, Grosskreutz J, Abrahams S, Bede P, Govind V, et al. A large-scale multicentre cerebral diffusion tensor imaging study in amyotrophic lateral sclerosis. J. Neurol. Neurosurg. Psychiatry 2016; 87: 570-579.

Neumann M, Sampathu DM, Kwong LK, Truax AC, Micsenyi MC, Chou TT, et al. Ubiquitinated TDP43 in Frontotemporal Lobar Degeneration and Amyotrophic Lateral Sclerosis. Science (80-. ). 2006; 314: 130-133.

Nicolas A, Kenna KP, Renton AE, Ticozzi N, Faghri F, Chia R, et al. Genome-wide Analyses Identify KIF5A as a Novel ALS Gene. Neuron 2018; 97: 1268-1283.e6.

Pandya S, Mezias C, Raj A. Predictive model of spread of progressive supranuclear palsy using directional network diffusion. Front. Neurol. 2017; 8

Pandya S, Zeighami Y, Freeze B, Dadar M, Collins DL, Dagher A, et al. Predictive model of spread of Parkinson's pathology using network diffusion. Neuroimage 2019; 192: 178-194.

Philips T, Rothstein JD. Glial cells in amyotrophic lateral sclerosis. Exp. Neurol. 2014; 262: 111-120.

Polymenidou M, Cleveland DW. The seeds of neurodegeneration: prion-like spreading in ALS. Cell 2011; 147: 498-508.

Poudel GR, Harding IH, Egan GF, Georgiou-Karistianis N. Network spread determines severity of degeneration and disconnection in Huntington's disease. Hum. Brain Mapp. 2019; 40: 4192-4201.

Raj A, Kuceyeski A, Weiner M. A Network Diffusion Model of Disease Progression in Dementia [Internet]. Neuron 2012Available from:

https://www.ncbi.nlm.nih.gov/pmc/articles/PMC3623298/

Raj A, LoCastro E, Kuceyeski A, Tosun D, Relkin N, Weiner M. Network Diffusion Model of Progression Predicts Longitudinal Patterns of Atrophy and Metabolism in Alzheimer's Disease. Cell Rep. 2015; 10: 359-369.

Ravits J, Laurie P, Fan Y, Moore DH. Implications of ALS focality: Rostral-caudal distribution of lower motor neuron loss postmortem. Neurology 2007a; 68: 1576-1582.

Ravits J, Paul P, Jorg C. Focality of upper and lower motor neuron degeneration at the clinical onset 
of ALS. Neurology 2007b; 68: 1571-1575.

Ravits JM, La Spada AR. ALS motor phenotype heterogeneity, focality, and spread: Deconstructing motor neuron degeneration. Neurology 2009; 73: 805-811.

Riku Y. Reappraisal of the anatomical spreading and propagation hypothesis about TDP-43 aggregation in amyotrophic lateral sclerosis and frontotemporal lobar degeneration. In: Neuropathology. Blackwell Publishing; 2020.

Robberecht W, Eykens C. The genetic basis of amyotrophic lateral sclerosis: recent breakthroughs. Adv. Genomics Genet. 2015; 5: 327.

Schmidt R, de Reus MA, Scholtens LH, van den Berg LH, van den Heuvel MP. Simulating disease propagation across white matter connectome reveals anatomical substrate for neuropathology staging in amyotrophic lateral sclerosis. Neuroimage 2016; 124: 762-769.

Scotter EL, Chen H-J, Shaw CE. TDP-43 Proteinopathy and ALS: Insights into Disease Mechanisms and Therapeutic Targets. Neurotherapeutics 2015; 12: 352-363.

Seeley WW, Crawford R, Rascovsky K, Kramer JH, Weiner M, Miller BL, et al. Frontal paralimbic network atrophy in very mild behavioral variant frontotemporal dementia. Arch. Neurol. 2008; 65: 249-55.

Seeley WW, Crawford RK, Zhou J, Miller BL, Greicius MD. Neurodegenerative Diseases Target LargeScale Human Brain Networks. Neuron 2009; 62: 42-52.

Smith BN, Topp SD, Fallini C, Shibata H, Chen H-J, Troakes C, et al. Mutations in the vesicular trafficking protein annexin A11 are associated with amyotrophic lateral sclerosis. [Internet]. Sci. Transl. Med. 2017; 9Available from: http://www.ncbi.nlm.nih.gov/pubmed/28469040 Spillantini MG, Crowther RA, Jakes R, Hasegawa M, Goedert M. $\alpha$-Synuclein in filamentous inclusions of Lewy bodies from Parkinson's disease and dementia with Lewy bodies. Proc. Natl. Acad. Sci. U. S. A. 1998; 95: 6469-6473.

Subramaniam S. Selective neuronal death in neurodegenerative diseases: The ongoing mystery. Yale J. Biol. Med. 2019; 92: 695-705.

Talbot K, Feneberg E, Scaber J, Thompson AG, Turner MR. Amyotrophic lateral sclerosis: the complex path to precision medicine. J. Neurol. 2018; 265: 2454-2462. 
Tu S, Menke RAL, Talbot K, Kiernan MC, Turner MR. Regional thalamic MRI as a marker of widespread cortical pathology and progressive frontotemporal involvement in amyotrophic lateral sclerosis. J. Neurol. Neurosurg. Psychiatry 2018; 89: 1250-1258.

Turner MR, Bowser R, Bruijn L, Dupuis L, Ludolph A, McGrath M, et al. Mechanisms, models and biomarkers in amyotrophic lateral sclerosis. Amyotroph. Lateral Scler. Front. Degener. 2013; 14: $19-32$.

Turner MR, Brockington A, Scaber J, Hollinger H, Marsden R, Shaw PJ, et al. Pattern of spread and prognosis in lower limb-onset ALS. Amyotroph. Lateral Scler. 2010; 11: 369-373.

Turner MR, Swash M. The expanding syndrome of amyotrophic lateral sclerosis: a clinical and molecular odyssey. J. Neurol. Neurosurg. Psychiatry 2015; 86: 667-673.

Vajda A, McLaughlin RL, Heverin M, Thorpe O, Abrahams S, Al-Chalabi A, et al. Genetic testing in ALS: A survey of current practices. Neurology 2017; 88: 991-999.

Verstraete E, Veldink JH, van den Berg LH, Van den Heuvel MP. Structural brain network imaging shows expanding disconnection of the motor system in amyotrophic lateral sclerosis. Hum. Brain Mapp. 2014; 35: 1351-1361.

Verstraete E, Veldink JH, Mandl RC, van den Berg LH, van den Heuvel MP. Impaired structural motor connectome in amyotrophic lateral sclerosis. PLoS One 2011; 6: e24239.

Walker LC, Diamond MI, Duff KE, Hyman BT. Mechanisms of protein seeding in neurodegenerative diseases. JAMA Neurol. 2013; 70: 304-10.

Westeneng H-J, Verstraete E, Walhout R, Schmidt R, Hendrikse J, Veldink JH, et al. Subcortical structures in amyotrophic lateral sclerosis. Neurobiol. Aging 2015; 36: 1075-1082. 


\section{Supplementary Information}
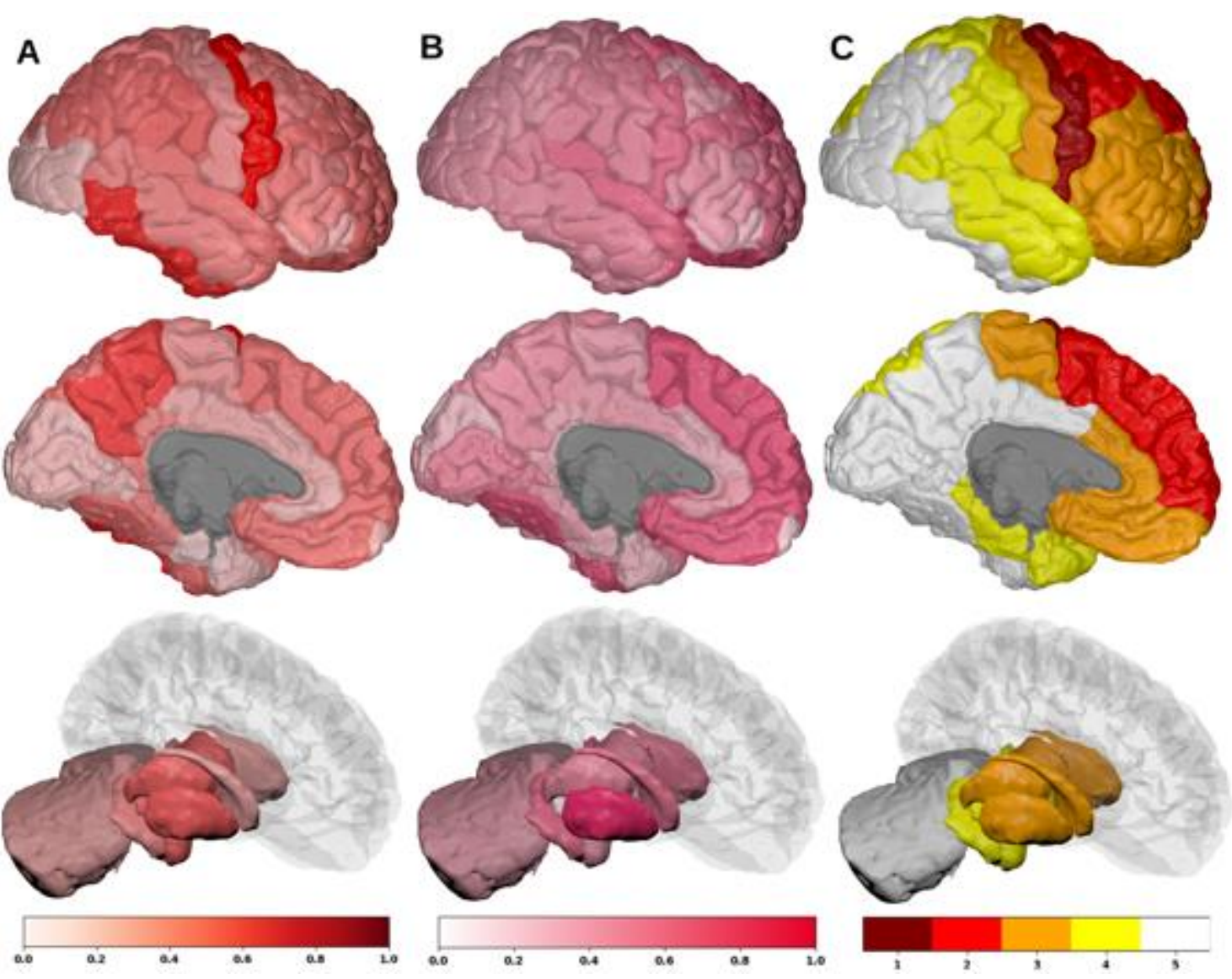

Figure SI - 5: Spatial distribution of ALS atrophy, NDM Predicted atrophy and histopathological staging. A] Measured regional ALS atrophy are depicted by "glass brain" visualization. Bilateral volume loss is observed in somatosensory, frontotemporal, and subcortical regions, with most atrophy occurring in precentral gyrus, inferior temporal gyrus, precuneus, putamen, and thalamic regions. Severity of disease in each region is depicted in a color bar, where color towards red show increased severity. B] Glass brains of NDM seeded at the bilateral thalamus at $t_{\max }=8.08$ au yields progression of ALS from thalamus to connected extra-motor, subcortical and frontal areas. Bilateral volume loss is mainly observed in frontal and subcortical regions, with most atrophy occurring in insula, putamen, later orbito-frontal, superior frontal, and fusiform. Severity of disease in each region is depicted in a color bar, where color towards magenta showing increased severity. C] The ALS stage from 1-4 for each of the 43 bilateral regions. We can see that Stage 1 which is indicated by maroon starts with agranular motor cortex. The next affected regions (stage 2 in red) are the premotor cortex and parts of prefrontal neocortex. The pathology then progresses into striatum and into the prefrontal/postcentral cortices (stage 3 in orange), and finally to stage 4 show in yellow involving anteromedial portions of the temporal lobe and the hippocampus. Stage 5 denotes regions in white that are not part of ALS staging schema (i.e. outside the 4 accepted stages in ALS). 

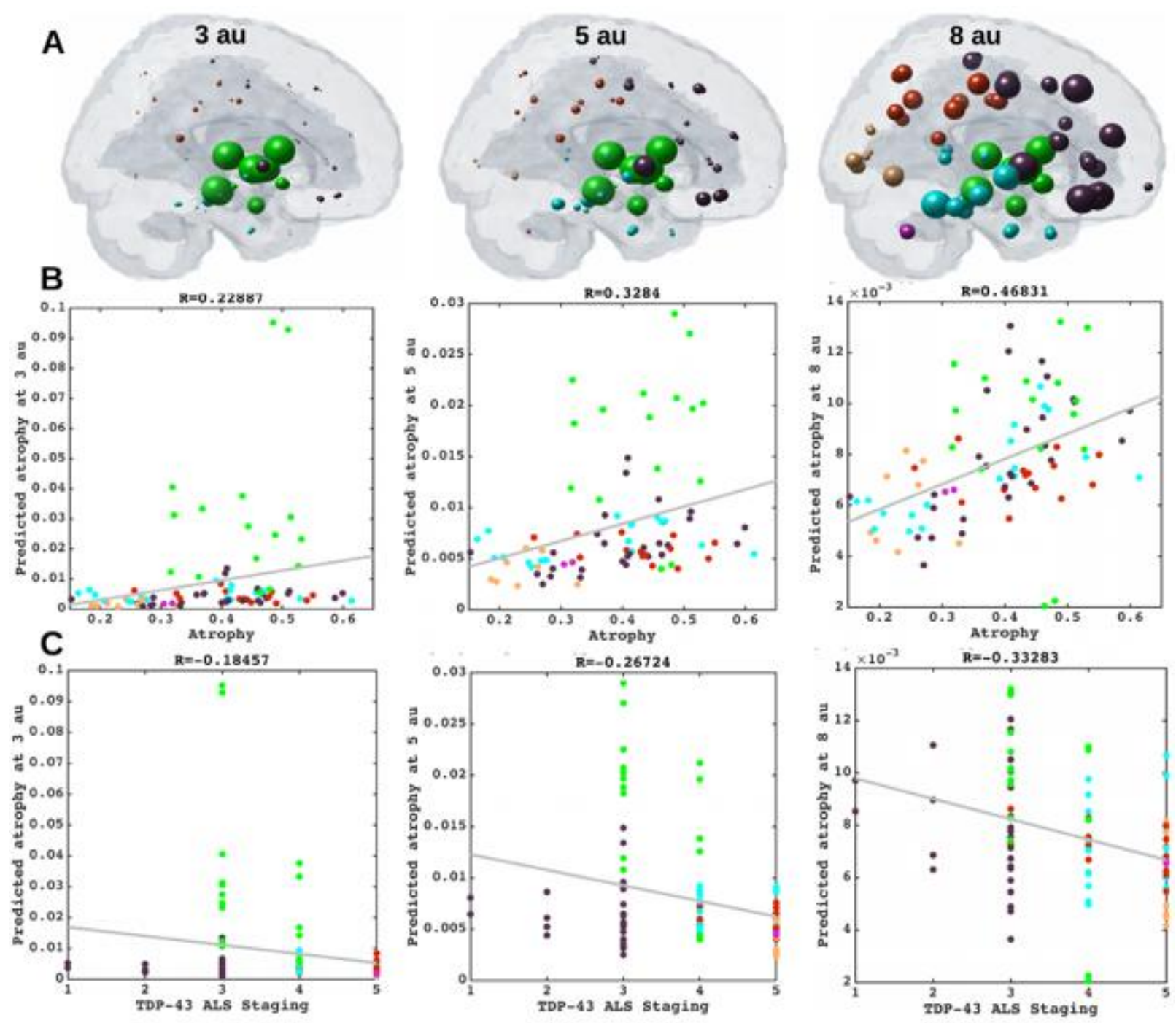

Figure SI - 6: Spatiotemporal evolution, scatter plots of atrophy and histopathological staging with NDM at different model times. A] Evolution of thalamic-seeded network diffusion at model times $t=3,5,8$ (au) exhibits subcortical areas as early affected regions, followed by somewhat slower diffusion into the motor and extra-motor cortices, specially prefrontal, and finally showing widespread involvement of cortical regions. This temporal sequencing predicted by the model suggest that volume loss in ALS involves extra-motor regions, particularly the prefrontal and subcortical regions. B] Scatter plot of NDM from thalamus versus empirical ALS atrophy at model times $t=3,5,8$ (au). Dots are color coded by lobe - frontal = purple; parietal = red; occipital = orange; temporal = cyan; subcortical = green; and cerebellum = magenta . A positive correlation is observed between ALS empirical atrophy and NDM predicted atrophy from bilateral thalamus, which increases significantly $\left(R=0.47, p_{\text {corr }}<5.8 \times 10^{-4}\right.$, $0.05 / 86)$ at matured model times $t=5,8$ (au). As time progresses, more and more frontal, temporal and extra-motor regions are involved with NDM closely resembling ALS atrophy. C] Scatter plot of NDM from thalamus versus ALS staging of TDP-43 pathology at model times $t=3,5,8$ (au). Dots are color coded by lobe - frontal = purple; parietal = red; occipital = orange; temporal = cyan; subcortical = green; and cerebellum = magenta. A negative correlation is observed between the NDM and ALS staging from bilateral thalamus, which decreases significantly $\left(R=-0.33, p_{\text {corr }}<5.8 \times 10^{-4}\right)$ at matured model times $t=$ 
bioRxiv preprint doi: https://doi.org/10.1101/2021.02.24.431118; this version posted February 25, 2021. The copyright holder for this preprint (which was not certified by peer review) is the author/funder, who has granted bioRxiv a license to display the preprint in perpetuity. It is made available under aCC-BY 4.0 International license.

5,8 (au). As time progresses, more and more frontal, temporal and subcortical regions are involved with NDM closely resembling empirical ALS atrophy.
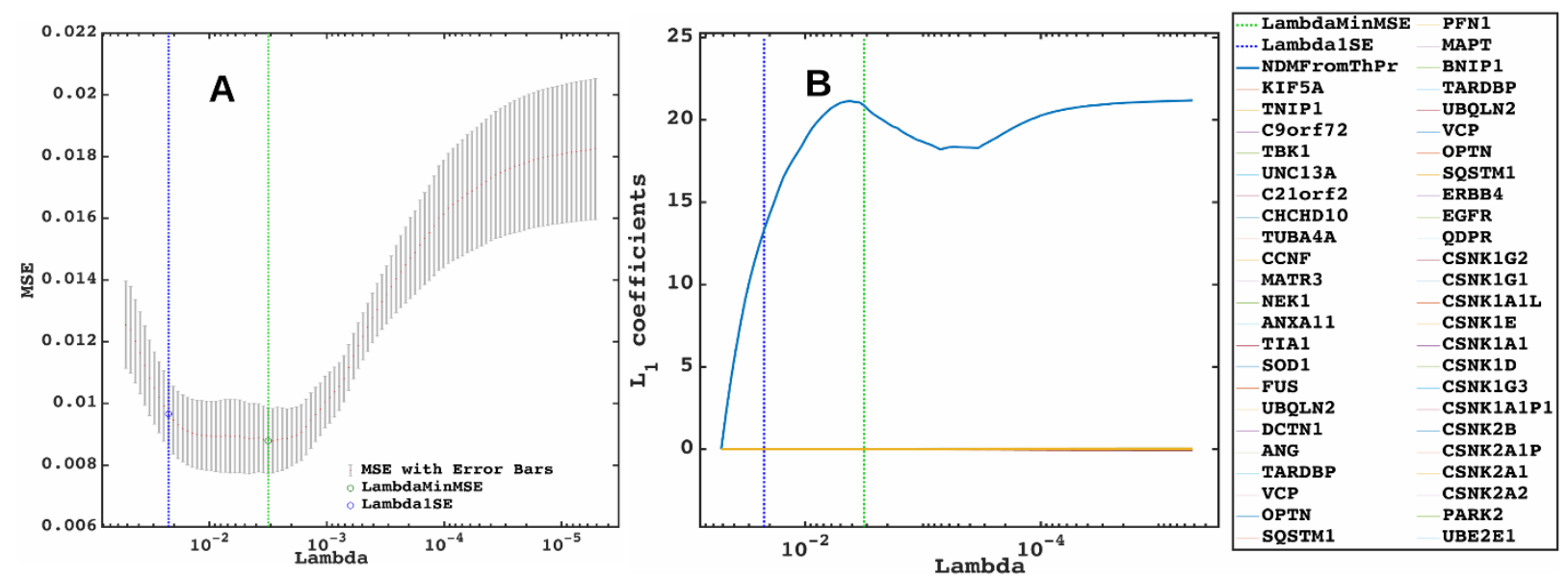

\begin{tabular}{|c|c|c|c|c|}
\hline Region Names & Estimate & SE & tstat & p-val \\
\hline NDM & 17.703 & 4.52 & 3.91 & 0.0002 ** \\
\hline KIF5A & -0.04 & 0.01 & -2.76 & 0.0075 * \\
\hline TBK1 & 0.04 & 0.02 & 2.16 & 0.0346 * \\
\hline TIA1 & 0.04 & 0.01 & 3.15 & 0.0025 * \\
\hline BNIP1 & 0.03 & 0.01 & 3.11 & 0.0028 * \\
\hline CSNK1G1 & -0.05 & 0.02 & -2.48 & 0.0158 * \\
\hline CSNKID & -0.04 & 0.02 & -2.18 & 0.0330 * \\
\hline CSNK2A1 & 0.04 & 0.01 & 2.79 & 0.0068 * \\
\hline CSNK2A1P & -0.03 & 0.01 & -2.09 & 0.0409 * \\
\hline CSNK2A2 & 0.04 & 0.02 & 2.13 & 0.0367 * \\
\hline ** $\mathrm{P}_{\text {corr }}<9.6 \times 10^{-4} ; * p<0.05$ & & & & \\
\hline
\end{tabular}

Figure SI - 7: Lasso plots and model parameters. A] Ten-fold cross validated MSE curves for determining regularized parameter lambda. Predictors with minimum L1 coefficient as a function of regularized parameter lambda with no more than one standard deviation (blue dotted line) were considered to be the most favorable. B] Cross-validated $L_{1}$ regularized regression coefficients as a function of tuning parameter lambda for a model containing the NDM from thalamus, ALS-related genes, and genes implicated in trans-synaptic TDP-43 transfer as predictors. Trace plot shows that's as lambda increases towards the left, lasso sets various coefficients to zero, thereby removing them from the model. C] Model parameters and p-values of significant predictors that survived with $p<0.05$ (represented with "*") and with Bonferroni corrected p (represented with "**"). The NDM and expression profiles of KIF5A, TBK1, TIA1, BNIP1, CSNK1G1, CSNKID, CSNK2A1, CSNK2A1P, and CSNK2A2 have nonzero coefficients at minimum model MSE, indicating that these are essential predictive variables. 

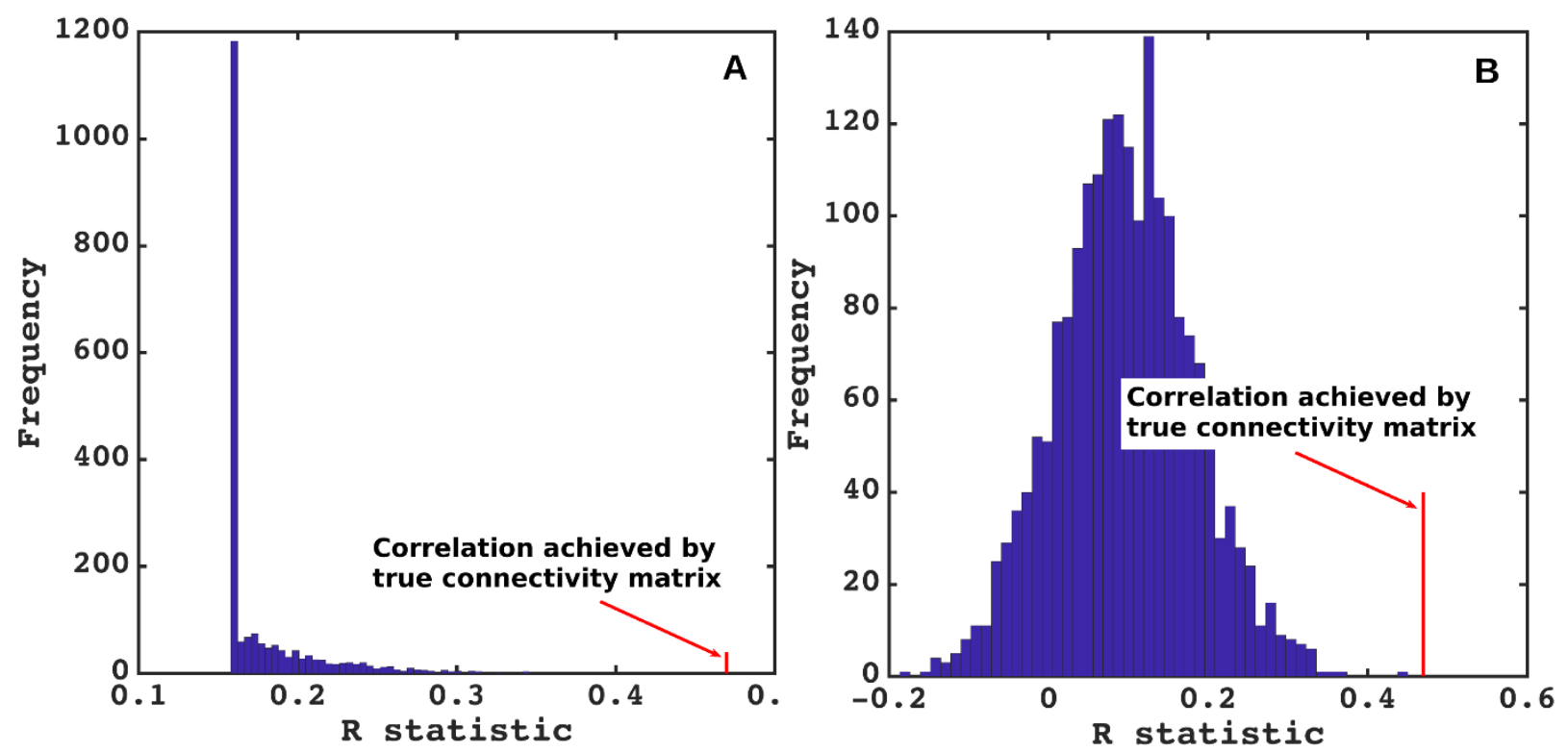

Figure SI - 8: NDM evaluation against alternate models. A] Histogram of correlation strength between NDM and ALS data over 2000 shuffled networks. There is a hard limit on the left of this plot at $R \sim 0.15$, which corresponds to the zero-diffusion time value of curve in Figure 1D. B] Histogram of correlation strength between NDM and 2000 shuffled ALS data over using unshuffled structural connectome. The true connectome was shuffled by symmetrically permuting its rows and columns randomly, and the NDM was evaluated for each shuffled network after bilateral thalamic-seeding. The best $R$ achieved by each model was recorded and entered into the histogram. The null models are distributed well below the true model, indicating that the latter is highly unlikely to arise by chance ( $p$ $<0.05)$. 
bioRxiv preprint doi: https://doi.org/10.1101/2021.02.24.431118; this version posted February 25, 2021. The copyright holder for this preprint (which was not certified by peer review) is the author/funder, who has granted bioRxiv a license to display the preprint in perpetuity. It is made available under aCC-BY 4.0 International license.

Table SI - 1: Empirical atrophy of top 20 regions (average of both left and right hemispheres)

\begin{tabular}{|c|c|}
\hline Region Names & Average empirical atrophy \\
\hline Precentral & 0.5929 \\
\hline Inferiortemporal & 0.5717 \\
\hline Precuneus & 0.5451 \\
\hline Putamen & 0.5099 \\
\hline ThalamusProper & 0.4972 \\
\hline Amygdala & 0.4918 \\
\hline Lateralorbitofrontal & 0.4843 \\
\hline Supramarginal & 0.4804 \\
\hline Pallidum & 0.4790 \\
\hline Hypothalamus & 0.4714 \\
\hline Rostralmiddlefrontal & 0.4621 \\
\hline Inferiorparietal & 0.4614 \\
\hline Fusiform & 0.4599 \\
\hline Superiorfrontal & 0.4509 \\
\hline Superiorparietal & 0.4431 \\
\hline Parsopercularis & 0.4425 \\
\hline Superiortemporal & 0.4420 \\
\hline Medialorbitofrontal & 0.4414 \\
\hline Caudalmiddlefrontal & 0.4239 \\
\hline Middletemporal & 0.4124 \\
\hline
\end{tabular}




\begin{tabular}{|c|c|}
\hline Region Names from structural connectome & $\mathbf{R}_{\max }$ \\
\hline ThalamusProper & 0.4694 \\
\hline Insula & 0.4508 \\
\hline Pallidum & 0.4422 \\
\hline Putamen & 0.4196 \\
\hline Caudate & 0.3977 \\
\hline Accumbensarea & 0.3928 \\
\hline Parsopercularis & 0.3924 \\
\hline Lateralorbitofrontal & 0.3923 \\
\hline Parstriangularis & 0.392 \\
\hline Medialorbitofrontal & 0.392 \\
\hline Parsorbitalis & 0.3904 \\
\hline Rostralmiddlefrontal & 0.3903 \\
\hline Precentral & 0.3898 \\
\hline Caudalmiddlefrontal & 0.3897 \\
\hline Frontalpole & 0.3864 \\
\hline Superiorfrontal & 0.3846 \\
\hline Rostralanteriorcingulate & 0.3836 \\
\hline Caudalanteriorcingulate & 0.379 \\
\hline Postcentral & 0.3643 \\
\hline Paracentral & 0.3453 \\
\hline Maximum Pearson Correlation & \\
\hline Pis & \\
\hline
\end{tabular}


bioRxiv preprint doi: https://doi.org/10.1101/2021.02.24.431118; this version posted February 25, 2021. The copyright holder for this preprint (which was not certified by peer review) is the author/funder, who has granted bioRxiv a license to display the preprint in perpetuity. It is made available under aCC-BY 4.0 International license.

Table SI - 3: Correlations of ALS-related genes, and their PCA vs empirical atrophy (to the left) and correlations of TDP-43 specific genes and their PCA vs empirical atrophy (to the right).

\begin{tabular}{|c|c|c|c|c|c|}
\hline \multicolumn{3}{|c|}{ All implicated genes } & \multicolumn{3}{|c|}{ TDP-43 genes } \\
\hline Gene Names & Pearson R & p-val & Gene Names & Pearson R & p-val \\
\hline KIF5A & -0.1656186196 & 0.1275200472 & TARDBP & 0.0587166421 & 0.5912596775 \\
\hline TNIP1 & -0.047536623 & 0.6638298672 & UBQLN2 & -0.1408213232 & 0.1959180963 \\
\hline C9orf72 & -0.2210541014 & 0.040202527 & VCP & $-1.99 E-01$ & $6.65 \mathrm{E}-02$ \\
\hline TBK1 & -0.0512079554 & 0.6396119229 & OPTN & 0.2324602021 & 0.0312558543 \\
\hline UNC13A & 0.0658038117 & 0.5471989112 & SQSTM1 & 0.046762839 & 0.6689795153 \\
\hline C21orf2 & 0.1303410162 & 0.2316356969 & ERBB4 & 0.1651074327 & 0.1287159075 \\
\hline CHCHD10 & 0.298541968 & 0.0052379237 & EGFR & -0.0495807645 & 0.6503011148 \\
\hline TUBA4A & 0.1365186655 & 0.2100696947 & QDPR & 0.1376219042 & 0.206373713 \\
\hline CCNF & 0.1614933579 & 0.1374160643 & CSNK1G2 & -0.2607516048 & 0.0153154119 \\
\hline MATR3 & -0.2673955129 & 0.0128125354 & CSNK1G1 & -0.1431117999 & 0.1886707366 \\
\hline NEK1 & -0.0228555052 & 0.834542713 & CSNK1A1L & 0.0437732909 & 0.6890175914 \\
\hline ANXA11 & -0.1058693483 & 0.3319691099 & CSNK1E & -0.2381853029 & 0.0272160232 \\
\hline TIA1 & -0.3156106649 & 0.0030756285 & CSNK1A1 & -0.059771397 & 0.5846018753 \\
\hline SOD1 & 0.2477350679 & 0.0214620101 & CSNK1D & -0.032865878 & 0.7638668473 \\
\hline FUS & -0.0717255375 & 0.5116516682 & CSNK1G3 & -0.1775994452 & 0.1018558544 \\
\hline UBQLN2 & -0.1408213232 & 0.1959180963 & CSNK1A1P1 & 0.0813687883 & 0.4564062443 \\
\hline DCTN1 & 0.128764788 & 0.2373770587 & CSNK2B & 0.2155003034 & 0.0462938926 \\
\hline ANG & -0.0558291315 & 0.6096588566 & CSNK2A1P & 0.0962652913 & 0.3779312784 \\
\hline TARDBP & 0.0587166421 & 0.5912596775 & CSNK2A1 & -0.0830659204 & 0.4470364806 \\
\hline VCP & -0.1988299645 & 0.0664668969 & CSNK2A2 & 0.201434283 & 0.0629134834 \\
\hline OPTN & 0.2324602021 & 0.0312558543 & PARK2 & -0.0576630116 & 0.5979443426 \\
\hline SQSTM1 & 0.046762839 & 0.6689795153 & UBE2E1 & 0.1597739631 & 0.1417082943 \\
\hline PFN1 & 0.383757023 & 0.0002648659 & UBE2E2 & -0.0478000201 & 0.6620804566 \\
\hline MAPT & 0.07828104 & 0.4737282635 & UBE2E3 & 0.184386267 & 0.0892228173 \\
\hline BNIP1 & 0.1176351583 & 0.2807256022 & USP14 & -0.2344893188 & 0.0297697703 \\
\hline PCA1 & 0.1797 & 0.0979 & USP8 & 0.0853785204 & 0.4344434894 \\
\hline \multirow[t]{2}{*}{ PCA2 } & 0.0515 & 0.6375 & PCA1 & 0.0625 & 0.5676 \\
\hline & & & PCA2 & 0.1850 & 0.0881 \\
\hline
\end{tabular}

